\title{
Acupuncture alleviates chronic pain and comorbid conditions in a mouse model of neuropathic pain: the involvement of DNA methylation in the prefrontal cortex
}

\author{
Jae-Hwan Jang ${ }^{a, b}$, Eun-Mo Song ${ }^{c}$, Young-Ho Do ${ }^{d}$, Sora Ahn ${ }^{a, b}$, Ju-Young Oh ${ }^{a, b}$, Tae-Yeon Hwang ${ }^{a, b}$, \\ Yeonhee Ryu ${ }^{\mathrm{e}}$, Songhee Jeon ${ }^{\mathrm{f}}$, Mi-Yeon Song ${ }^{\mathrm{c}}$, Hi-Joon Park ${ }^{\mathrm{a}, \mathrm{b}, *}$
}

\begin{abstract}
Chronic pain reduces life quality and is an important clinical problem associated with emotional and cognitive dysfunction. Epigenetic regulation of DNA methylation is involved in the induction of abnormal behaviors and pathological gene expression. We examined whether acupuncture can restore epigenetic changes caused by chronic pain, and identified the underlying mechanisms in neuropathic pain mice. Acupuncture treatment for 6 months (3 days/week) improved mechanical/cold allodynia and the emotional/cognitive dysfunction caused by left partial sciatic nerve ligation (PSNL)-induced neuropathic pain. The effects of acupuncture were associated with global DNA methylation recovery in the prefrontal cortex (PFC). Analysis of DNA methylation patterns in PFC indicated that 1364 overlapping genes among 4442 and 4416 methylated genes in the PSNL vs sham and PSNL vs acupuncture points groups, respectively, were highly associated with the DNA methylation process. Acupuncture restored the reduced expression of 5-methylcytosine, methyl-cytosine-phospho-guanine binding protein 2, and DNA methyltransferase family enzymes induced by PSNL in PFC. Methylation levels of Nr4a1 and Chkb associated with mitochondrial dysfunction were decreased in PFC of the PSNL mice, and increased by acupuncture. By contrast, high expression of Nr4a1 and Chkb mRNA in PSNL mice decreased after acupuncture. We also found that acupuncture inhibited the expression of Ras pathway-related genes such as Rasgrp1 and Rassf1. Finally, the expression of Nr4a1, Rasgrp1, Rassf1, and Chkb mRNA increased in the neuronal cells treated with Mecp2 small interfering RNA. These results suggest that acupuncture can relieve chronic pain-induced comorbid conditions by altering DNA methylation of Nr4a1, Rasgrp1, Rassf1, and Chkb in the PFC.
\end{abstract}

Keywords: Acupuncture, Comorbid condition, Prefrontal cortex, Epigenetic, DNA methylation

\section{Introduction}

Chronic pain, defined as persistent pain for at least 3 to 6 months, is associated with comorbidities, such as emotional and cognitive impairment, which further adversely affect quality of life. ${ }^{6,38,64,68,71}$ The ineffectiveness of current therapies, at least, partly results from insufficient understanding of the various mechanisms underlying chronic pain. Thus, clarifying the factors that influence chronic pain, including physiological mechanisms involving the central nervous system (CNS), will help develop novel treatments.
Chronic neuropathic pain results from pathological adaptations in the CNS associated with brain structure ${ }^{3,19,60,61,66}$ and connectivity alterations $\mathrm{s}^{8,77}$ in the prefrontal cortex (PFC), $3,61,66$ hippocampus (HIP), ${ }^{3}$ amygdala (AMG), 66,77 hypothalamus, ${ }^{19}$ and periaqueductal gray matter (PAG) ${ }^{8,77}$ Numerous chronic pain studies have reported that PFC is related to pain and comorbidity modulation. ${ }^{4,13-15}$ A preclinical rodent study showed that induction of peripheral nerve injury leads to gray matter reduction and longterm changes in gene expression in the PFC; these factors are involved in chronic pain or associated behaviors. ${ }^{1,2,55,60}$ However,

\footnotetext{
Sponsorships or competing interests that may be relevant to content are disclosed at the end of this article.

${ }^{a}$ Acupuncture and Meridian Science Research Center (AMSRC), Kyung Hee University, Dongdaemun-gu, Seoul, Republic of Korea, ${ }^{b}$ Department of Korean Medical Science, Graduate School of Korean Medicine, Kyung Hee University, Dongdaemoon-gu, Seoul, Republic of Korea, ${ }^{c}$ Department of Physical Medicine and Rehabilitation, Graduate School of Korean Medicine, Kyung Hee University, Dongdaemun-gu, Seoul, Republic of Korea, ${ }^{d}$ Department of Psychiatry, Medical Science Research Institute, Dongguk University Medical Center, Goyang, Republic of Korea, ${ }^{e}$ Korea Institute of Oriental Medicine, Yuseong-gu, Daejeon, Republic of Korea, ${ }^{f}$ Department of Biomedical Sciences, Center for Creative Biomedical Scientists at Chonnam National University, Gwangju, Republic of Korea
}

${ }^{*}$ Corresponding author. Address: Acupuncture and Meridian Science Research Center (AMSRC), College of Korean Medicine, Kyung Hee University, 26 Kyungheedae-ro, Dongdaemun-gu, Seoul 02447, Republic of Korea. Tel.: +82 2-961-9435. E-mail address: acufind@khu.ac.kr (H.J. Park).

Supplemental digital content is available for this article. Direct URL citations appear in the printed text and are provided in the HTML and PDF versions of this article on the journal's Web site (www. painjournalonline.com).

PAIN 162 (2021) 514-530

Copyright (0) 2020 The Author(s). Published by Wolters Kluwer Health, Inc. on behalf of the International Association for the Study of Pain. This is an open access article distributed under the terms of the Creative Commons Attribution-Non Commercial-No Derivatives License 4.0 (CCBY-NC-ND), where it is permissible to download and share the work provided it is properly cited. The work cannot be changed in any way or used commercially without permission from the journal.

http://dx.doi.org/10.1097/j.pain.0000000000002031 
the mechanisms underlying the changes in the PFC associated with chronic pain comorbidities are not yet clearly understood.

Epigenetic modifications of gene expression in response to environmental changes are reversible and dynamic. ${ }^{66}$ DNA methylation is associated with synaptic plasticity and memory formation, and is a critical epigenetic mechanism underlying alterations in gene expression without affecting DNA sequences. ${ }^{12,24}$ DNA methylation partly silences gene expression through recruitment of methyl-cytosine-phospho-guanine (CpG) binding protein 2 (MeCP2), which selectively associates with methylated CpG dinucleotides mediated by DNA methyltransferases (DNMT). ${ }^{44}$ DNA methylation plays a role in oncology, neurological diseases, pathological gene expression states, and abnormal activity in the CNS. ${ }^{42,43,45}$ DNA methylation also plays an important role in chronic pain. ${ }^{12,13,33,35}$ Mouse studies indicate that neuropathic pain is accompanied by comorbidity that persists for several months after injury, and decreased global DNA methylation has been detected in the PFC of mice in neuropathic pain. ${ }^{22,66}$

Acupuncture, practiced for more than 2500 years in East Asia, has been used to treat chronic pain and emotional and cognitive disorders. ${ }^{62,63,70,78}$ Both clinical and preclinical studies have indicated that acupuncture improves chronic pain comorbidities. ${ }^{7,20,25}$ In our previous studies, we demonstrated that acupuncture restores both mechanical hyperalgesia and comorbid cognitive impairment by modulating synaptic plasticityrelated protein expression levels in the HIP of a partial sciatic nerve ligation (PSNL) mouse mode ${ }^{26}$ and exhibits neuroprotective effects in a Parkinson disease mouse model induced by mitochondrial dysfunction. ${ }^{32,50}$ However, how acupuncture affects the expression of these genes is unclear. Recent studies have shown that acupuncture modulates the epigenetic mechanism in the brain. ${ }^{11,76}$

We hypothesized that DNA methylation alterations in the brain are involved with chronic pain and comorbidities, which may be ameliorated by acupuncture. In this study, we investigated whether acupuncture improved allodynia, negative emotional states, and reduced cognitive capacity in mice 6 months after PSNL. DNA methylation in pain-related brain areas and possible pathways underlying the effects of acupuncture were also explored.

\section{Materials and methods}

\subsection{Animals}

Seven-week-old male C57BL/6J mice (weight, 22-25 g; Samtaco, Osan, South Korea) were housed individually at $24 \pm 2^{\circ} \mathrm{C}$ under a 12/12-hour light/dark cycle (light: 08:00-20:00, dark: 20: 00-08:00) with free access to food and water for at least 7 days before the commencement of the experiments. All the experimental protocols were approved by the Dongguk University Animal Care Committee for Animal Welfare (IACUC-2017-022-1).

\subsection{Neuropathic pain model}

A left hind paw PSNL model was established in accordance with the approach of Malmberg and Basbaum et al. ${ }^{40}$ with some modifications. Mice were anesthetized with $1 \%$ Rompun (100 $\mu \mathrm{L}$, intraperitoneally [i.p.]; Bayer, Seoul, South Korea) and 2\% Zoletil (100 $\mu$ L, i.p.; Virbac S.A., Carros, France). The bilateral hind thighs were shaved, and the sciatic nerve was exposed using scissors. The dorsal $1 / 3$ to $1 / 2$ of the nerve was then lightly ligated with 8-0 silk (Ailee, Busan, South Korea), and the wound was closed. In the Sham (non-nerve-injured) group, the nerve was exposed (and closed) without injury. Animals were randomly allocated to groups, and preacupuncture testing was conducted at least 7 days after PSNL.

\subsection{Acupuncture and positive control treatment}

Eight days after each PSNL surgery, acupuncture treatment was applied at the GB30 (Hwando)/GB34 (Yanglingquan) acupuncture points (AP) 3 days/week (Monday, Wednesday, and Friday) for 6 months. The GB30 is located at the junction of the lateral 1/3 and medial $2 / 3$ of the greater trochanter with the sacral hiatus, and GB34 is located in the depression anterior and distal to the head of the fibula (Fig. 1A). These acupoints have been used for the treatment of lumbar neuropathic pain in clinical studies. ${ }^{9}$ The combination of those acupoints has been used to treat neuropathic pain such as sciatica, which includes aching and sharp pain components that radiate from the buttock downward along the course of the sciatic nerve. ${ }^{17,57}$ These acupoints were selected by a process of consensus with doctors of Korea medicine who all specialized in Rehabilitation Medicine of Korean Medicine on the basis of a literature review regarding acupuncture for low back pain and sciatica. ${ }^{31}$ For the acupuncture treatments, mice were mildly immobilized, and a sterilized acupuncture needle $(8 \mathrm{~mm}$ in length and $0.18 \mathrm{~mm}$ in diameter; Haenglim-seoweon Acuneedle Co, Gyeonggi-Do, South Korea) was inserted bilaterally to a depth of $3 \mathrm{~mm}$ and turned bidirectionally for 30 seconds (twice per second; one $180^{\circ}$ clockwise rotation and one $180^{\circ}$ counterclockwise rotation) using the fingers. Using hematoxylin and eosin staining, we observed that there were needle tracts around the acupunctured sites regardless of acupoint but no tissue damage and/or inflammatory response (data not shown). To exclude the nonspecific effects of acupuncture, the same acupuncture stimulation and procedure ( 3 days/week for 6 months) were applied to the control points (CP group). One is located on the lateral part of biceps femoris $1 \mathrm{~cm}$ distal from the gluteal fold and the other is located on the gluteus maximus $5 \mathrm{~mm}$ lateral from the base of the tail (Fig. 1A).

As a positive control (PC), amitriptyline (10 mg/kg in $100 \mu \mathrm{L}$; Sigma-Aldrich, St. Louis, MO) was administered intraperitoneally (i.p.) 3 days/week for 6 months, from day 8 after PSNL surgery. A solution of amitriptyline was freshly prepared in $0.9 \% \mathrm{NaCl}$ on each treatment day. To ensure equal stress conditions among groups, the animals in the Sham, PSNL, and PC groups were also mildly immobilized, as in the AP and CP groups.

\subsection{Behavioral tests}

\subsubsection{Nociceptive behavioral tests}

Mechanical hyperalgesia of the left hind paws was assessed using electronic von Frey filaments (IITC, Woodland Hills, CA). Before the experiment, the mice were habituated to a clear $8 \times 10$ $\times 10 \mathrm{~cm}$ acrylic box with a gridded floor, as used in the actual experiment. ${ }^{27}$ The habituation took place over 1 hour each day, for 2 days. Baseline mechanical hyperalgesia was assessed at least 1 day before PSNL surgery. The mice were placed back into the same clear box, and the mechanical thresholds were assessed. The von Frey filament was applied to the plantar surface of each hind paw of each mouse 10 times, using a force of $0.6 \mathrm{~g}$ and with a 5-sec interval between each application. ${ }^{27}$ The number of positive responses to the 10 applications was counted. Mechanical hyperalgesia was defined as an increase in paw withdrawal frequency in response to nonpainful 
stimulation. ${ }^{27}$ The baseline measurement was performed 1 day before the PSNL surgery. The mice were then randomly assigned to 5 groups; paw withdrawal frequency did not differ among the groups. The effects of treatments with acupuncture (AP or CP) and amitriptyline in the PSNL-induced neuropathic pain model were investigated for 6 months (0 day, 1 week, 2 weeks, and 1-6 months).

Cold allodynia of the left hind paw was assessed using the acetone test. The total lick duration (flinching, licking, or biting) of acetone-evoked behaviors was measured within a 1-minute period after spraying acetone $(\sim 20 \mu \mathrm{L})$ onto the plantar surface of the hind paw with a syringe.

\subsubsection{Anxiety-like behavioral tests}

Anxiety-like behaviors were evaluated using the open-field test (OFT) and elevated plus-maze (EPM) test at 6 months after PSNL. For OFT, after habituation for approximately 1 hour in the experimental room, each mouse was individually housed in a rectangular white box $(40 \times 40 \times 27 \mathrm{~cm})$ in a dimly lit room $(23 \pm$ 1 lux). This box provided the best contrast to the black mice. A video camera was installed above the box in the center of the room, and the behaviors of the mice (number of times they crossed the central zone and total distance travelled) were recorded for 5 minutes and monitored using a computerized video-tracking system running the S-MART program (Pan Lab Co, Barcelona, Spain).

The EPM test was used to evaluate approach-avoidance behavior in mice. After habituation to the experimental room for 1 hour, the mice were allowed to individually explore the maze (arm length, $16 \mathrm{~cm}$; arm width, $5 \mathrm{~cm}$; closed arm height, $12 \mathrm{~cm}$ ) for 5 minutes, and their behavior was recorded using a video camera mounted on the ceiling above the center of the maze using the S-MART program. The mice explored the open arms of the maze under indirect, dim light (23 \pm 1 lux). After each mouse had explored the apparatus, the maze was washed with 70\% alcohol. At the beginning of each trial, mice were placed in such a way that they were facing a closed arm at the center of the maze.

\subsubsection{Depression-like behavioral tests}

The tail suspension test (TST) was used to measure depressionlike behavior. Mice were individually suspended by the tail on a horizontal bar (40 cm from the floor) using masking tape placed roughly $4 \mathrm{~cm}$ from the tip of the tail. The immobility time was recorded in seconds over a period of 6 minutes and defined as the absence of escape-oriented behavior.

\subsubsection{Cognitive function tests}

Cognitive function tests were measured using the novel object recognition (NOR) and $\mathrm{Y}$-maze test at 6 months after PSNL operation. Spontaneous alternation in the $Y$-maze was tested using the following protocol: each mouse was placed in arm of the $Y$-maze and was free to explore the maze for 5 minutes. ${ }^{49}$ The total number of entered arms and the order of entry were recorded. Arm entry was considered complete when the tail was entirely within the arm. The percentage of spontaneous alternation was determined as follows: number of trials including entry into all 3 arms/maximum possible number of alternations (total number of arms entered - 2) $\times 100 .{ }^{49}$

The NOR test was used to evaluate short-term memory. The apparatus consisted of a square area $(40 \times 40 \times 27 \mathrm{~cm})$ constructed in polyvinyl chloride with a black floor and walls for mice, and a round arena (diameter: $50 \mathrm{~cm}$ ) with a white floor and walls for mice. ${ }^{23}$ The box and objects therein were cleaned between trials to remove residual olfactory cues. Animals underwent 2 habituation sessions (5 minutes each) in the empty box; 24 hours later, each mouse was first placed in the box and exposed to 2 same familiar objects for 5 minutes. After 24 hours,

A

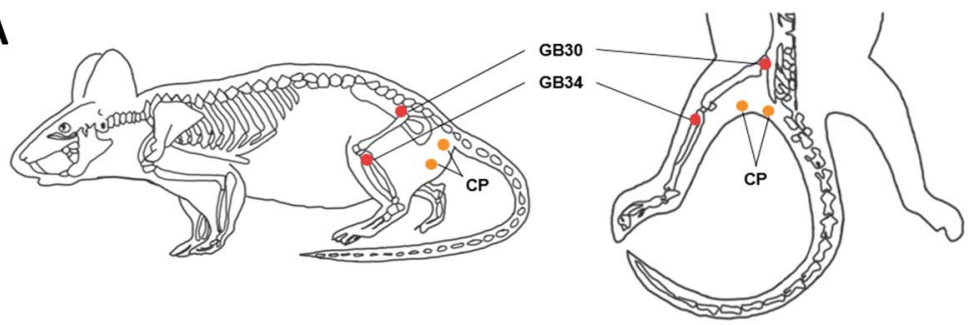

B

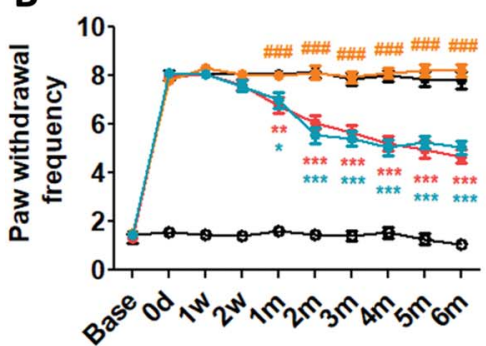

C

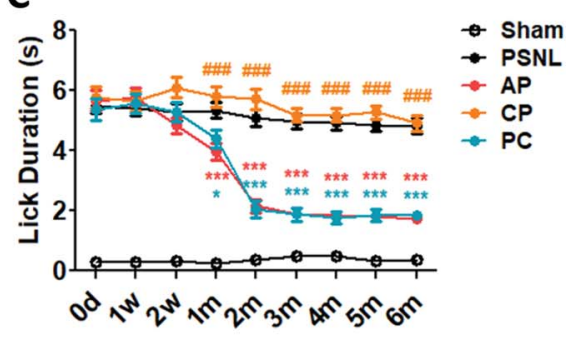

Figure 1. Effects of acupuncture on mechanical hyperalgesia and cold allodynia, evaluated using the von Frey and acetone tests. The effects of acupuncture treatment (acupoints GB30 [Hwando] and GB34 [Yanglingquan]; AP group), stimulation of nonacupoints (CP group) (A), and amitriptyline (PC group, 10 mg/kg, intraperitoneally [i.p.]) applied 3 days/week for 6 months on mechanical hyperalgesia and cold allodynia were investigated using the von Frey (B) and cold (acetone) (C) tests in a partial sciatic nerve ligation (PSNL)-induced neuropathic pain model. The antiallodynic effects of AP, CP, and PC (10 mg/kg, i.p.) were measured on the ipsilateral plantar surfaces 2 hours after administration (for 0, 1, and 2 weeks, and 1-6 months) ( $\mathrm{n}=22 / \mathrm{group}$. ${ }^{\star} P<0.05$, ${ }^{\star \star} P<0.01$, ${ }^{\star \star \star} P<0.001 \mathrm{vs}$ the $\mathrm{PSNL}$ group, and \#\#\#P < 0.001 vs the AP group). Data were analyzed using two-way repeated-measures analysis of variance (ANOVA) followed by Bonferroni post hoc test. The results are expressed as the mean \pm SEM. AP, acupuncture points; CP, control point; PC, positive control. 
the one familiar object was replaced by a novel object. The animal was then placed back in the box and exposed to the familiar object, and to a novel test object, for a further 5 minutes. The time spent exploring each object was measured. The recognition index was calculated as the ratio of time spent exploring the novel object to the total exploration time, reflecting short-term memory capacity.

\subsection{Enzyme-linked immunosorbent assay}

The frontal cortex, including the prelimbic and intraorbital regions, was extracted from mice at 6 months after PSNL surgery, frozen on dry ice, and stored at $-80^{\circ} \mathrm{C}$ until use. Genomic DNA was extracted using a DNeasy Blood \& Tissue Kit (Qiagen, Hilden, Germany). The lysate was first passed through a DNA spin column to selectively isolate DNA. DNA was extracted using a modified version of the column-based method described by Bettscheider et al. ${ }^{75}$ DNA quantity and quality were then assessed using the NanoDrop 2000 instrument (Thermo Scientific, Wilmington, $\mathrm{DE})$; the $\mathrm{OD}_{260} / \mathrm{OD}_{280}$ ratio was $\sim 1.8$ for all samples. Global DNA methylation was performed using a Methylflash Global DNA Methylation 5-methylcytosine (5-mC) enzyme-linked immunosorbent assay Easy Kit (P-1030; Epigentek, Farmingdale, NY) in accordance with the manufacturer's instructions. Briefly, methylated DNA was detected using capture and detection antibodies, to 5-mC, and then measured colorimetrically by reading the absorbance at $450 \mathrm{~nm}$ using a VersaMax Microplate reader (Molecular Devices, San Jose, CA).

\subsection{Whole-genome bisulfite sequencing}

The integrity of the gDNA was checked by running an agarose gel electrophoresis and gDNA was quantified using Quant-IT PicoGreen (Invitrogen, Carlsbad, CA). The whole-genome bisulfite sequencing (WGBS) libraries were prepared using Accel-NGS Methyl-Seq DNA library kit (Swift BioSciences, Ann Harbor, MI). Briefly, fragmentation of $200 \mathrm{ng}$ of gDNA was performed using the Covaris LE220 focused-ultrasonicator (Covaris, Woburn, MA) to a target peak size of 400 to $700 \mathrm{bp}$. The fragmented DNA were then subject to bisulfite conversion using the EZ DNA Methylation-Gold Kit (Zymo Research, Orange, CA), according to manufacturer's instructions. The bisulfite-treated ssDNA fragments were repaired and the truncated adapter 1 and 2 ligated to the $3^{\prime}$ end and $5^{\prime}$ end of fragments, respectively. The truncated adapter-ligated DNA was amplified with an indexed primer to complete the WGBS libraries with full-length adapter. The final libraries were then quantified using qPCR according to the qPCR Quantification Protocol Guide (KAPA Library Quantification kits for Illumina Sequencing platforms) and qualified using the TapeStation DNA screentape D1000 (Agilent). And then we sequenced using the HiSeq platform (Illumina, San Diego, CA). Paired-end sequencing reads (151 bp) generated from WGBS were verified of their sequence quality with Fast QC (version 0.11.7). Before starting analysis, TrimGalore (version 0.5.0) was used to remove adapter sequences. The cleaned reads were aligned to the mus musculus genome (mm10) using Bisulfite Sequence Mapping Program (BSMAP) based on the SOAP (Short Oligo Alignment program). BSMAP (version 2.90 parameter set - r $0-n$ 1) allowing up to (read length $\times 8 \%$ ) nucleotide mismatches to the reference genome per read and returning only uniquely mapped reads. Mapped data (SAM file format) were performed sorting and indexing using SAM tools (version 1.9). Then, PCR duplicates were removed with SAMBAMBA (version 0.6.5). Methylation level was called with the methratio.py in
BSMAP program. ${ }^{72}$ For the regions covered by both ends of a read pair, only one read is used to call methylation. The resulting coverage profiles are summarized as \# of C/effective CT counts for each of the 3 sequence contexts ( $\mathrm{CG}, \mathrm{CHG}$, and $\mathrm{CHH})$.

For each CpG, 7,574,123 CpGs outside the lower read and higher read cutoffs in total CpG methylation sites of 43,735,674 are excluded leaving 36,161,551 CpGs to be analyzed. Lower read cutoff of 10 means that bases with coverage below 10X are discarded because a high-enough read coverage will increase the power of the statistical tests. Higher read cutoff of 500 means that bases with more than 500X in each sample are discarded. Methylation level at each base of filtered CpG was normalized with median scaling normalization. We applied an independent $T$ test to assess significance of methylation differences between 2 groups for 3 comparison pairs. Differentially methylated CpGs were determined by filtering for each region associated with I delta_meanl $\geq 0.2$ and independent $T$ test $P$-values $<0.05$. Hierarchical clustering analysis also was performed using complete linkage and Euclidean distance as a measure of similarity to display the methylation level of samples for significant CpGs that are satisfied with ldelta_meanl $\geq 0.2$ and independent $T$ test $P$-values $<0.05$ in comparison pair. Gene-enrichment analysis and Kyoto Encyclopedia of Genes and Genomes (KEGG) pathway analysis for annotated gene with differentially methylated $\mathrm{CpGs}$ on promoter region, which is defined upstream $2 \mathrm{~kb}$ region from transcription start site, were also performed based on Gene Ontology (GO) (http://geneontology.org/) and KEGG pathway (https://www.genome.jp/kegg/) database, respectively. All statistical analyses and visualization of differentially expressed genes were conducted using R.3.5.1 (www.r-project.org).

\subsection{Reverse transcription-PCR}

Total RNA from the PFC area of brain in Sham, PSNL, and AP groups (each $n=3$ ) was isolated using TRIzol reagent (Invitrogen). Isolated RNA $(2 \mu \mathrm{g})$ was incubated for 60 minutes at $42^{\circ} \mathrm{C}$ using SuperScript III First-Strand (Invitrogen), and amplified using KAPA Taq PCR kit (Kapa Biosystems, Wilmington, MA) according to the manufacturer's instructions and using the primers. All primer sequences' information is provided in Table 1. The cDNA samples after polymerase chain reaction (PCR) were separated on a 1\% agarose gel, and stained with GelRed (Biotium, Fremont, CA). The density of sample band was measured using an ImageJ program, and was normalized on intensity of $\beta$-actin.

\subsection{Quantitative reverse transcription-PCR}

Real-time PCR was performed using LightCycler Nano Instrument (Roche Molecular Systems, Inc) with KAPA SYBR FAST qPCR Master Mix (Kapa Biosystems) according to the manufacturer's instructions. All primer sequence-related information is provided in Table 1. The results of $\beta$-actin mRNA were used as for normalization and as an internal control probe.

\subsection{Immunofluorescence}

Immunofluorescence was conducted ${ }^{27}$ to measure the expression of $5-\mathrm{mC}, \mathrm{MeCP} 2$ in neuron cells in the PFC. Primary antibodies raised against NeuN (mouse, 1:500, MAB377; Chemicon International, Inc, Temecula, CA), 5-mC (rabbit, 1: 500, \#28692; Cell Signaling Technology, Beverly, MA), MeCP2 (rabbit, 1:1,000, \#3456; Cell Signaling Technology), and brainderived neurotrophic factor (mouse, 1:10, sc-65514; Santa Cruz 
Biotechnology, Santa Cruz, CA) were diluted in $1 \times$ PBST(phosphate buffered saline (PBS) $+0.3 \%$ Triton X-100) supplemented with $0.3 \%$ bovine serum albumin. The plates were wrapped in aluminum foil to block light and then stored at $4^{\circ} \mathrm{C}$ for 72 hours. The tissue sections were sequentially incubated for 1 hour with a mixture of Alexa Fluor 488-conjugated donkey anti-mouse secondary antibody (1:1000; Thermo Fisher Scientific, Fremont, CA) and Alexa Fluor 594-conjugated donkey anti-goat secondary antibody (1:2000; Thermo Fisher Scientific). Cell counting (NeuN/ $5-\mathrm{mC}$ and/MeCP2) was performed using a square grid $(300 \times$ $300 \mu \mathrm{m})$ in the PFC.

\subsection{Western blotting}

Western blot was performed ${ }^{51}$ to measure the protein expression levels of DNMTs in the PFC. Primary antibodies were rabbit DNMT1 (1:1000, \#5032; Cell Signaling Technology), DNMT3a (1: 1000, \#2160; Cell Signaling Technology), DNMT3b (1:2000, NB300-516; Novusbio, Littleton, CO), brain-derived neurotrophic factor (1:500, sc-546; Santa Cruz Biotechnology), and mouse $\beta$-actin (1:10,000, A1978; Sigma-Aldrich). The membranes were then incubated with secondary horseradish peroxidaseconjugated goat anti-rabbit antibody (1:2000; Pierce, Rockford, IL) and anti-mouse antibody (1:30,000 for $\beta$-actin, Pierce). Signals were visualized using a chemiluminescence kit (Super Signal West Pico; Pierce) and intensity was measured by densitometry.

\subsection{Primary cortical neuron cultures and Mecp2 small interfering RNA treatment}

Frontal cortical neurons were isolated from a male C57BL/6J mouse (5 weeks, Samtaco) that was anaesthetized using ether. The isolated cortex was chopped and transferred to $9 \mathrm{~mL}$ Hanks balanced salt solution and was incubated in 10X trypsin (1 mL) at $37^{\circ} \mathrm{C}$ for 20 minutes, and was then neutralized in trypsin inhibitor. Single neuronal cells dissociated from the whole frontal cortex of the mouse were plated on poly-D-lysine precoated 6-well plates $\left(2.0 \times 10^{6}\right.$ cells/well). Cell cultures were incubated in the neurobasal medium with 2\% B27 supplement, $0.2 \mathrm{mM}$ glutamine, $0.1 \mathrm{mg} / \mathrm{mL}$ streptomycin, and $100 \mathrm{U} / \mathrm{mL}$ penicillin, and were maintained at $37^{\circ} \mathrm{C}$ under a humidified $5 \% \quad \mathrm{CO}_{2}$ atmosphere. $^{37}$

To investigate the effects of Mecp2 small interfering RNA (siRNA) on gene expression in the frontal cortex, the neuronal cells were pretreated with the Mecp2 siRNA (5 pmol/well, sc35893; Santa Cruz Biotechnology; Mecp2 siRNA group) or control siRNA (5 pmol/well, sc-37007; Santa Cruz Biotechnology; Control group) before 3 days, and then, RNA was extracted from those cells using TRIzol reagent (Invitrogen). Reverse transcription-PCR and quantitative RT-PCR were performed for measuring the mRNA expression of Mecp2, Nr4a1, Rasgrp1, Rassf1, and Chkb.

\subsection{Statistical analyses}

All statistical analyses were performed using GraphPad Prism 5.0 software (GraphPad Software, San Diego, CA). Anxiety- and depression-like behaviors based on the OFT and EPM and TST, cognitive dysfunction based on the Y-maze and NOR tests, Western blotting, enzyme-linked immunosorbent assay, quantitative RT-PCR analysis, and immunofluorescence data were subjected to one-way analysis of variance (ANOVA) followed by Newman-Keuls tests. Analyses of mechanical hyperalgesia and cold allodynia at various time points were performed using twoway repeated-measures ANOVA, with Bonferroni post hoc test for pairwise multiple comparisons. Spearman rank correlation

Table 1

Primer sequences and condition for RT-PCR analysis.

\begin{tabular}{|c|c|c|c|}
\hline Primer & Sequence & & Condition \\
\hline Mecp2 (mouse) & $\begin{array}{l}\text { Forward } \\
\text { Reverse }\end{array}$ & $\begin{array}{l}\text { 5'-TGGTAAAACCCGTCCGGAAA-3' } \\
5^{\prime} \text {-TTCCTCCCTGAGCCCTAACA-3' }\end{array}$ & $58^{\circ} \mathrm{C}$ \\
\hline Dnmt1 (mouse) & $\begin{array}{l}\text { Forward } \\
\text { Reverse }\end{array}$ & $\begin{array}{l}\text { 5'-TGTTCTGTCGTCTGCAACCT-3' } \\
\text { 5'-AACTAGGTGAAGTTTCAAAAAGGGT-3' }\end{array}$ & $58^{\circ} \mathrm{C}$ \\
\hline Dnmt3a (mouse) & $\begin{array}{l}\text { Forward } \\
\text { Reverse }\end{array}$ & $\begin{array}{l}\text { 5'-TGAGCTGTACTGCAGAGGGG-3' } \\
\text { 5'-TTCGTAGATGGCTTTGCGGT-3' }\end{array}$ & $55^{\circ} \mathrm{C}$ \\
\hline Dnmt3b (mouse) & $\begin{array}{l}\text { Forward } \\
\text { Reverse }\end{array}$ & $\begin{array}{l}\text { 5'-GCCAGACCTTGGAAACCTCA-3' } \\
5^{\prime} \text {-GGCTGGAGACCTCCCTCTTA-3' }\end{array}$ & $58^{\circ} \mathrm{C}$ \\
\hline Nr4a1 (mouse) & $\begin{array}{l}\text { Forward } \\
\text { Reverse }\end{array}$ & $\begin{array}{l}\text { 5'-GAAAGTTGGGGGAGTGTGCTA-3' } \\
\text { 5'-GGTGTCAAACTCTCCGGTGT-3' }\end{array}$ & $60^{\circ} \mathrm{C}$ \\
\hline Rasgrp1 (mouse) & $\begin{array}{l}\text { Forward } \\
\text { Reverse }\end{array}$ & $\begin{array}{l}\text { 5'-ATGAGGGCCAGCTCCATCTA-3' } \\
\text { 5'-ATCCTTCTTCGGGTGCATGG-3' }\end{array}$ & $59^{\circ} \mathrm{C}$ \\
\hline Rassf1 (mouse) & $\begin{array}{l}\text { Forward } \\
\text { Reverse }\end{array}$ & $\begin{array}{l}\text { 5'-GAGCCAGAACTCATTGAACTACG-3' } \\
\text { 5'-TCATCCACGTTCGTATCCCG-3' }\end{array}$ & $59^{\circ} \mathrm{C}$ \\
\hline Chkb (mouse) & $\begin{array}{l}\text { Forward } \\
\text { Reverse }\end{array}$ & $\begin{array}{l}\text { 5'-TATCAGTGGTGCCGGGAGTA-3' } \\
5^{\prime} \text {-GAGAATGGCGAACATCACGC-3' }\end{array}$ & $59^{\circ} \mathrm{C}$ \\
\hline Abcb6 (mouse) & $\begin{array}{l}\text { Forward } \\
\text { Reverse }\end{array}$ & $\begin{array}{l}\text { 5'-GCGTTTATGCCGAGATGTGG-3' } \\
\text { 5'-TGCAGTACCCCACACAACC-3' }\end{array}$ & $59^{\circ} \mathrm{C}$ \\
\hline Tekt4 (mouse) & $\begin{array}{l}\text { Forward } \\
\text { Reverse }\end{array}$ & $\begin{array}{l}\text { 5'-TGCTCCATTGATCGTGCCTG-3' } \\
\text { 5'-GACGTGTAGGCGCCAGTATT-3' }\end{array}$ & $60^{\circ} \mathrm{C}$ \\
\hline$\beta$-actin (mouse) & $\begin{array}{l}\text { Forward } \\
\text { Reverse }\end{array}$ & $\begin{array}{l}\text { 5'-ACTCCTATGTGGGTGACGAG-3' } \\
\text { 5'-CATCTTTTCACGGTTGGCCTTAG-3' }\end{array}$ & $55^{\circ} \mathrm{C}$ \\
\hline
\end{tabular}


coefficient test was conducted to analyze whether the 5-mC expression levels in the brain regions (PFC, PAG, hypothalamus, $\mathrm{HIP}$, and AMG) were correlated with von Frey, acetone, OFT, EPM, Y-maze, NOR, and TST test results. Quantitative RT-PCR analysis in the Mecp2 siRNA treated-neuronal cell was subjected to unpaired 2-tailed $t$-tests. All data are expressed as the mean \pm SEM. In all analyses, $P<0.05$ was taken to indicate statistical significance.

\section{Results}

\subsection{Acupuncture improved persistent mechanical hyperalgesia and cold allodynia in partial sciatic nerve ligation-induced neuropathic pain}

The antimechanical and anticold allodynic effects of acupuncture were measured using the von Frey and acetone tests in a PSNLinduced neuropathic pain model. For mechanical allodynia, twoway repeated-measures ANOVA revealed a significant main effect of treatment $(F[4,1095]=520.0, P<0.0001)$ and a significant group $\times$ time interaction $(F[36,1095]=25.42, P<$ 0.0001). Bonferroni post hoc tests showed reversal of the established mechanical hyperalgesia at each time point in the AP group at all time points from 1 to 6 months after treatment $(P<$ 0.001 vs PSNL group; Fig. 1B). Similarly, the paw withdrawal frequency was significantly lower in the $\mathrm{PC}$ group at all time points from 1 to 6 months after treatment $(P<0.01$ or $P<0.001$ vs PSNL group; Fig. 1B). Regarding cold allodynia, two-way repeated-measures ANOVA revealed a significant main effect of treatment $(F[4,985]=181.8, P<0.0001)$ and a significant group $\times$ time interaction $(F[32,985]=21.51, P<0.0001)$. Bonferroni post hoc tests indicated that the total lick duration in the AP and PC groups was significantly lower than that in the PSNL group at all time points from 1 to 6 months after treatment (AP: all $P<$ 0.001 vs PSNL; PC: all $P<0.05$ or $P<0.001$ vs PSNL; Fig. 1C). Overall, the results showed that the acupoints had an analgesic effect.

\subsection{Acupuncture corrected persistent emotional disorders caused by partial sciatic nerve ligation}

We used OFT, EPM, and TST to examine the effects of acupuncture treatment on PSNL-induced emotional disorders. Regarding the OFT, one-way ANOVA showed a significant difference between groups in the number of crossings in the central zone $(F[4,105]=35.28, P<0.0001)$, and the NewmanKeuls post hoc test showed that the number of crossings in the central zone was higher in the AP group than in the PSNL and CP groups $(P<0.001$; Figs. $2 \mathrm{~A}$ and $\mathrm{B})$, and total distance did not differ in all groups (Fig. 2C). In the EPM test, one-way ANOVA ( $F$ $[4,105]=23.10, P<0.0001)$ followed by the Newman-Keuls post hoc test indicated that the PSNL-induced reduction in the number of entries into the open arms ( $P<0.001$ vs sham group) was restored by the AP and $\mathrm{PC}$ treatments (both $P<0.001$ vs PSNL group) and was improved in the AP group compared to the CP group (Figs. 2D and E). In addition, no group showed a significant change in the total distance traveled in EPM tests (Fig. 2F).

This study evaluated the antidepressant effects of acupuncture (AP or $\mathrm{CP}$ ) and amitriptyline (PC) on depression-like behaviors generated in a PSNL neuropathic pain model using the TST. Oneway ANOVA $(F[4,105]=50.97, P<0.0001)$ followed by the Newman-Keuls post hoc test showed a significant increase in immobility time in the PSNL group $(P<0.001$ vs Control), whereas AP treatment significantly reduced the immobility time $(P<0.001$ vs PSNL; Fig. 2G).

\subsection{Acupuncture restored persistent cognitive deficits caused by partial sciatic nerve ligation}

We used the $Y$-maze and NOR tests to examine the effects of acupuncture on cognitive impairment after PSNL surgery. Regarding the $\mathrm{Y}$-maze test, one-way ANOVA indicated a significant difference among all groups in spontaneous alternation $(F[4,105]=18.92, P<0.000)$, and the Newman-Keuls post hoc test indicated that AP treatment significantly improved spontaneous alternation in the $\mathrm{Y}$-maze test ( $P<0.05$ vs PSNL group; Figs. 3A and $\mathrm{B}$ ). Regarding the NOR test, one-way ANOVA revealed a significant difference among groups $(F[4,105]=25.87$, $P<0.0001$ ), and the Newman-Keuls post hoc test showed that the effects on cognition of repeated $A P$ and $P C$ treatments were significant compared to those in the PSNL group (both $P<0.001$; Figs. $3 \mathrm{C}$ and D).

\subsection{Acupuncture improved DNA methylation reduced in the prefrontal cortex, periaqueductal gray, hypothalamus, hippocampus, and amygdala after partial sciatic nerve ligation}

The effects of acupuncture (AP or CP; 3 days/week) for 6 months on DNA methylation in various brain areas in the PSNL-induced neuropathic pain model were examined. One-way ANOVA indicated a significant difference among all groups in DNA methylation in the PFC (F[3, 20] $=16.09, P<0.0001)$, and the Newman-Keuls post hoc test indicated that DNA methylation in the PSNL group was significantly reduced in the PFC $(P<0.001$ vs sham group). AP treatment significantly reversed global DNA methylation in the PFC ( $P<0.001$; Fig. 4A). In addition, Spearman rank correlation coefficient analyses were performed to investigate whether the changes in DNA methylation were correlated with changes in nociception, anxiety, depression, and cognitive function. Global DNA methylation in the PFC was negatively correlated with nociceptive and depression-like behaviors $(r=-0.7470, P=0.0052$ for von Frey test; $r=$ $-0.6713, P=0.0168$ for acetone test; $r=-0.6993, P=0.0114$ for TST), and positively correlated with anxiety-like behavior and cognitive dysfunction $(r=0.6702, P=0.0171$ for OFT; $r=$ $0.8693, P=0.0002$ for EPM; $r=0.6294, P=0.0283$ for $Y$-maze; $r=0.7719, P=0.0033$ for NOR; Figs. 4B-D; Figs. S1A-D, available online as supplemental digital content at http://links. Iww.com/PAIN/B143).

Significant difference was observed among the 4 groups in global DNA methylation in the PAG $(F[3,20]=5.780, P=$ $0.0051)$. DNA methylation in the PSNL group was significantly decreased in the PAG ( $P<0.05$ vs sham group). Global DNA methylation in the PFC was not significantly reversed by AP treatment (Fig. 4E). Global DNA methylation in the PAG was negatively correlated with nociceptive and depression-like behaviors $(r=-0.7399, P=0.0059$ for von Frey test; $r=$ $-0.6783, P=0.0153$ for acetone test; $r=-0.6434, P=0.0240$ for TST), and positively correlated with anxiety-like behaviors ( $r=$ $0.6281, P=0.0287$ for OFT; $r=0.5442, P=0.0674$ for EPM). However, DNA methylation in the PAG was not correlated with cognitive dysfunction $(r=0.2378, P=0.4586$ for $Y$-maze; $r=$ $0.4737, P=0.1198$ for NOR; Figs. 4F-H; Figs. S1E-H, available online as supplemental digital content at http://links.lww.com/ PAIN/B143). 
A
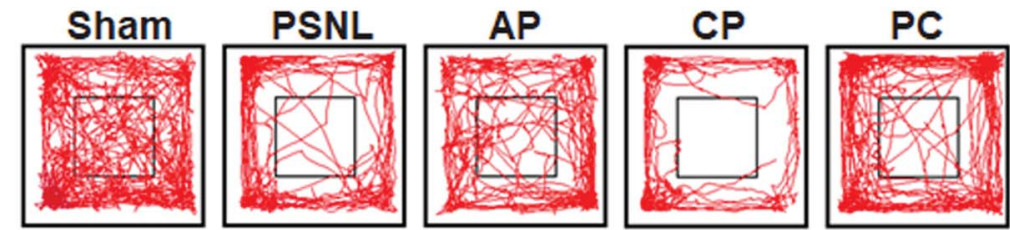

B

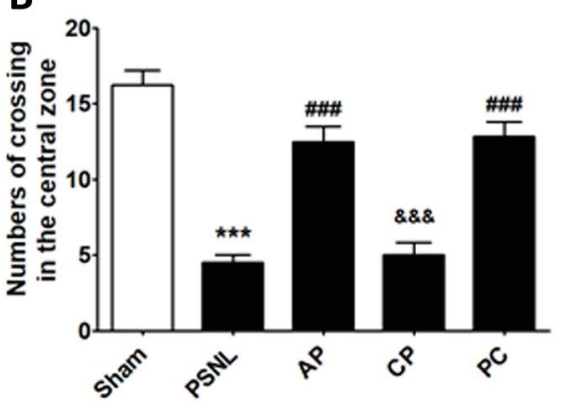

C

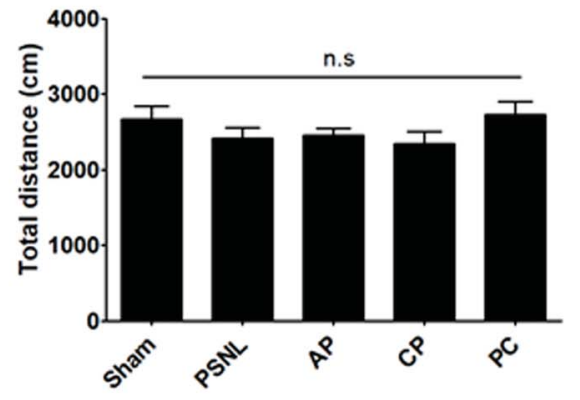

D
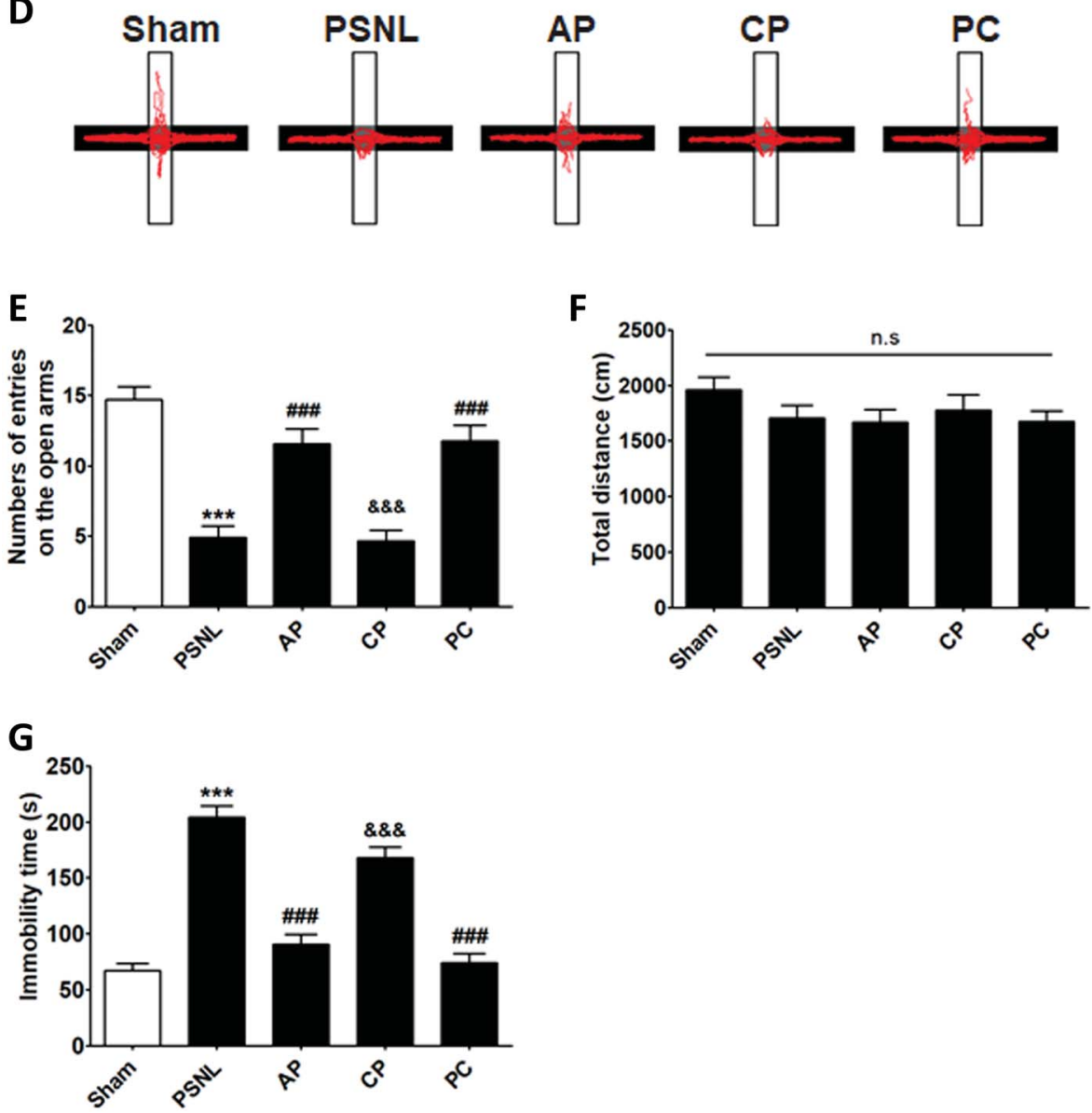

Figure 2. Effects of acupuncture on emotional functions measured using the OFT, EPM, and TST. The effects of acupuncture (AP and CP; 3 days/week for 6 months) and amitriptyline (PC, $10 \mathrm{mg} / \mathrm{kg}$, i.p.) administration on anxiety-like behaviors were demonstrated by OFT (A-C) and EPM (D-F) in a PSNL-induced neuropathic pain model. The effects of the various interventions on immobility time were measured using the TST (G); $n=22 /$ group. All data were analyzed using

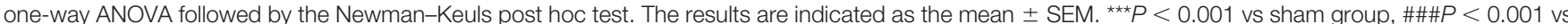
PSNL group, and \&\&\&P< 0.05 vs AP group. ANOVA, analysis of variance; AP, acupuncture points; CP, control point; EPM, elevated plus-maze; OFT, open-field test; PC, positive control; PSNL, partial sciatic nerve ligation; TST, tail suspension test.

Global DNA methylation in the hypothalamus ( $F[3,20]=4.792, P$ $=0.0113$ ) was not significantly increased in the PSNL group compared to the sham group, but was significantly decreased in the AP group ( $P<0.001$ vs PSNL group; Fig. 4I). DNA methylation in the hypothalamus was negatively correlated with anxiety-like behaviors $(r$ $=-0.5860, P=0.0453$ for OFT; $r=-0.5301, P=0.0763$ for EPM), and positively correlated with depression-like behaviors $(r=0.6364$, $P=0.0261$ for TST). However, DNA methylation in the hypothalamus 
A

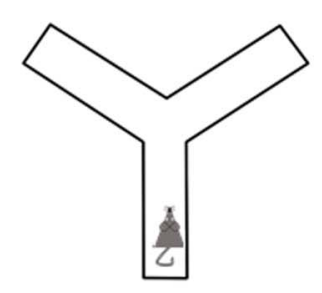

C

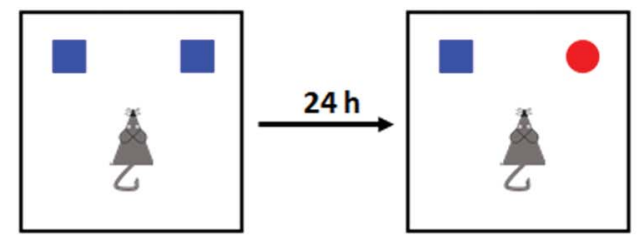

B
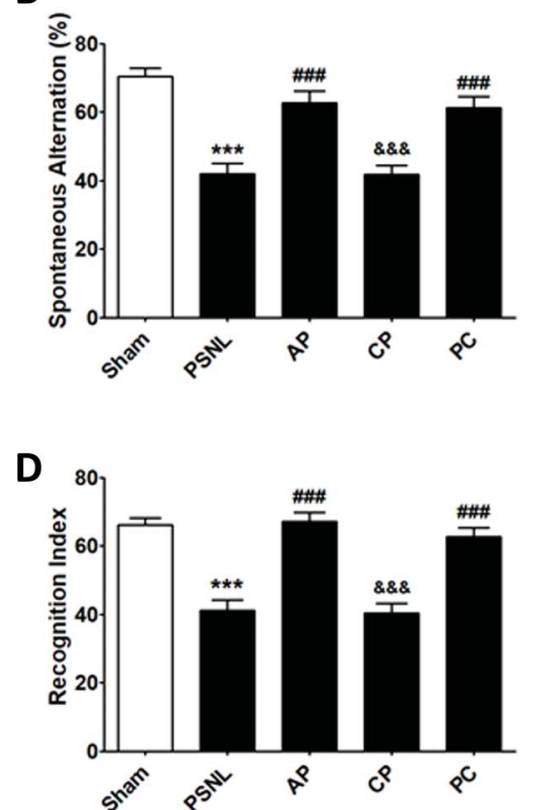

Figure 3. Effects of acupuncture on cognitive functions measured by Y-maze and NOR tests. Effects of acupuncture (AP and CP; 3 days/week for 6 months) and amitriptyline (PC, $10 \mathrm{mg} / \mathrm{kg}$, i.p.) treatment on cognitive impairment were examined using the Y-maze (A and B) and NOR (C and D) tests in a PSNL-induced

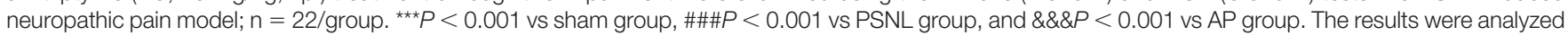
using one-way ANOVA followed by the Newman-Keuls post hoc test. All data are shown as the mean \pm SEM. ANOVA, analysis of variance; AP, acupuncture points; CP, control point; NOR, novel object recognition. PC, positive control; PSNL, partial sciatic nerve ligation.

was not correlated with nociceptive behaviors or cognitive dysfunction $(r=0.1947, P=0.5443$ for von Frey test; $r=0.1259, P=$ 0.6967 for acetone test; $r=-0.2308, P=0.4705$ for $Y$-maze; $r=$ $-0.3228, P=0.3061$ for NOR; Figs. 4J-L; Figs. S1I-L, available online as supplemental digital content at http://inks.Iww.com/PAIN/B143).

Significant difference was found among all the groups in global DNA methylation in the HIP $(F[3,20]=10.29, P=0.0003)$. Reduced DNA methylation after PSNL ( $P<0.001$ vs sham group) was improved by AP treatment $(P<0.01$ vs PSNL group; Fig. 4M). Global DNA methylation in the HIP was positively correlated with nociceptive and depression-like behaviors $(r=0.6655, P=$ 0.0182 for von Frey test; $r=0.5105, P=0.0899$ for acetone test; $r=0.8042, P=0.0016$ for TST), and negatively correlated with anxiety-like behavior and cognitive dysfunction $(r=-0.7439$, $P=0.0055$ for OFT; $r=-0.7103, P=0.0096$ for EPM; $r=$ $-0.4615, P=0.1309$ for $Y$-maze; $r=-0.6561, P=0.0205$ for NOR; Figs. 4N-P; Figs. S1M-P, available online as supplemental digital content at http://links.Iww.com/PAIN/B143).

DNA methylation in AMG did not show a significant difference between the 4 groups (AMG, F[3, 20] = 3.063, $P=0.0517$; Fig. $4 Q$ ). Global DNA methylation in the AMG was not correlated with any of the behaviors examined $(r=0.1274, P=0.6931$ for von Frey test; $r=0.1678, P=0.6021$ for acetone test; $r=0.4615$, $P=0.1309$ for TST; $r=-0.3228, P=0.3061$ for OFT; $r=$ $-0.3887, P=0.2117$ for EPM; $r=-0.3846, P=0.2170$ for Y-maze; $r=-0.2526, P=0.4282$ for NOR; Figs. 4R-T, Fig. S1Q - T, available online as supplemental digital content at http:// links.Iww.com/PAIN/B143).

\subsection{Acupuncture treatment induced DNA methylation pattern changes in the prefrontal cortex}

To investigate the acupuncture-specific methylated genes in the PFC, WGBS was performed to determine DNA methylation in
PFC areas isolated from the brains of mice. DNA methylation profiling in the promoter regions was performed to identify differentially methylated regions (DMRs) in the sham vs PSNL and PSNL vs AP group comparisons. Approximately 2451 hypermethylated genes and 1991 hypomethylated DMRs were selected from a total of 4442 genes in the PSNL vs sham group comparison (Fig. 5A), whereas 2217 hypermethylated genes and 2199 hypomethylated DMRs were selected from a total of 4416 genes in the PSNL vs AP group comparison (Fig. 5B). In addition, DMR analysis indicated that the methylation pattern in the PSNL group was distinct from that in the sham group (Fig. 5C), as well as from the AP group (Fig. 5D). Therefore, acupuncture resulted in DNA methylation alteration in the PFC after PSNL.

\subsection{Acupuncture affected DNA methylation process-related genes in the prefrontal cortex}

Differentially methylated regions in the promoter regions, which may affect the regulation of mRNA expression, were analyzed (Fig. 6A). Gene ontology analysis revealed high enrichments in processes related to DNA methylation $(P<0.05)$ such as "DNA metabolic process" (PSNL vs sham: $P=4.83 \times 10^{-15}, 128$ genes; PSNL Vs AP: $P=7.00 \times 10^{-22}, 146$ genes), "chromosomal part" (PSNL vs sham: $P=2.08 \times 10^{-14}, 126$ genes; PSNL vs AP: $P=1.51 \times 10^{-12}, 119$ genes), "chromatin binding" (PSNL vs sham: $P=2.41 \times 10^{-12}$, 88 genes; PSNL vs AP: $P=1.09 \times 10^{-10}, 83$ genes), "methylation" (PSNL vs sham: $P=3.45 \times 10^{-5}, 46$ genes; PSNL vs AP: $P=2.52 \times 10^{-6}, 49$ genes), and "C-5 methylation of cytosine" (PSNL vs sham: $P=$ $4.00 \times 10^{-2}$, 3 genes; PSNL vs AP: $P=3.91 \times 10^{-2}, 3$ genes; Fig. 6B). Kyoto Encyclopedia of Genes and Genomes pathway analysis indicated high enrichments in pathways related to metabolic and biological functions (protein synthesis, cell death, and memory) $(P<0.05)$ such as "metabolic pathways" (PSNL vs 



- Sham O PSNL $\bullet$ AP $\bullet$ CP

Figure 4. Effects of acupuncture on global DNA methylation levels in the PFC, PAG, hypothalamus, HIP, and AMG. Changes in global DNA methylation in the PFC (A), PAG (E), hypothalamus (I), HIP (M), and AMG (Q) after acupuncture (AP or $\mathrm{CP}$ ) for 6 months ( 3 days/week); $n=6 /$ group. ${ }^{*} P<0.05,{ }^{* *} P<0.01,{ }^{* \star *} P<0.001$ vs

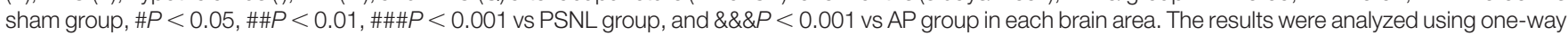
ANOVA followed by the Newman-Keuls post hoc test. The data are expressed as the mean \pm SEM. Representative graphs showing correlations between global DNA methylation levels in behavioral test results (von Frey test, EPM, and NOR) and each brain area: the PFC (B-D), PAG (E-H), hypothalamus (I-L), HIP (M-P), AMG (Q-T); $n=3$ /group. Spearman rank correlation coefficients were calculated. ANOVA, analysis of variance; AMG, amygdala; AP, acupuncture points; CP, control point; HIP, hippocampus; NOR, novel object recognition; PFC, prefrontal cortex; PAG, periaqueductal gray matter; PSNL, partial sciatic nerve ligation. 
sham: $P=2.40 \times 10^{-72}, 229$ genes; PSNL vs AP: $P=8.13 \times 10^{-84}$, 244 genes), "PI3K-Akt signaling pathway" (PSNL vs sham: $P=3.03$ $\times 10^{-74}, 74$ genes; PSNL vs AP: $P=6.35 \times 10^{-26}, 71$ genes), "MAPK signaling pathway" (PSNL vs sham: $P=1.24 \times 10^{-22}$, 61 genes; PSNL vs AP: $P=3.17 \times 10^{-19}, 55$ genes), "Ras signaling pathway" (PSNL vs sham: $P=1.56 \times 10^{-16}, 46$ genes; PSNL vs AP: $P=2.25 \times 10^{-20}, 51$ genes), and "MicroRNAs in cancer" (PSNL vs sham: $P=1.65 \times 10^{-14}, 47$ genes; PSNL vs AP: $P=4.85 \times 10^{-18}, 52$ genes; Fig. 6B).

To identify genes methylated in response to acupuncture treatment, we extracted 1364 overlapping genes in the PSNL vs sham (total of 4442 genes) and PSNL vs AP (total of 4416 genes) group comparisons (Fig. 6C). Overlapping genes (1364) shared Mecp2, Dnmt1, Dnmt3a, and Dnmt3b associated with DNA methylation in DNA metabolic processes (146) and metabolic pathways (244) (Fig. 6D). Because Mecp2 and Dnmt familial genes are known as the major players in the DNA methylation process, ${ }^{15,16,29}$ we investigated whether methylation and mRNA expression in these genes showed a different pattern. One-way ANOVA showed a significant difference among groups in DNA methylation in the promoter regions of the Mecp2 and Dnmt familial genes (Mecp2, F[2, 6] = 5.645, $P=0.0418$; Dnmt1, F[2, $6]=6.115, P=0.0357$; Dnmt3a, $\mathrm{F}[2,6]=20.43, P=0.0021$; Dnmt3b, F[2, 6] $=5.012, P=0.0525)$, and the Newman-Keuls post hoc test indicated that DNA methylation in the PSNL group was increased in the PFC (Mecp2, $P>0.05$; Dnmt1, $P>0.05$; Dnmt3a, $P<0.01$; Dnmt3b, $P>0.05$ vs each sham group), and was lower in the AP vs the PSNL group (Mecp2, $P<0.05$; Dnmt1, $P<0.05$; Dnmt3a, $P<0.01$; Dnmt3b, $P>0.05$ vs each PSNL group; Figs. 6E-H). Furthermore, we investigated mRNA expression levels of Mecp2 and Dnmt familial genes in the PFC. One-way ANOVA indicated a significant difference among groups in the mRNA levels of Mecp2 and Dnmt familial genes (Mecp2, F $[2,6]=2.648, P=0.1498 ;$ Dnmt1, F[2, 6] = 11.52, $P=0.0088$; Dnmt3a, F[2, 6] = 13.43, $P=0.0061 ;$ Dnmt3b, F[2, 6] $=3.276, P$ $=0.1092)$, and the Newman-Keuls post hoc test indicated that acupuncture improved mRNA expression levels that were reduced in the PSNL group (Mecp2, $P>0.05$; Dnmt1, $P<$ 0.01 ; Dnmt3a, $P<0.01$; Dnmt3b, $P>0.05$ vs each PSNL group; Figs. 6I-M). These results indicated that various genes, including Mecp2, Dnmt1, and Dnmt3a as well as those involved in the DNA methylation process, were restored by acupuncture.

\subsection{Acupuncture reversed 5-mC, MeCP2, and DNMT expression changes in the prefrontal cortex after PSNL}

To confirm the 5-mC and MeCP2 expression levels in the PFC neuron after acupuncture treatment (3 days/week for 6 months),
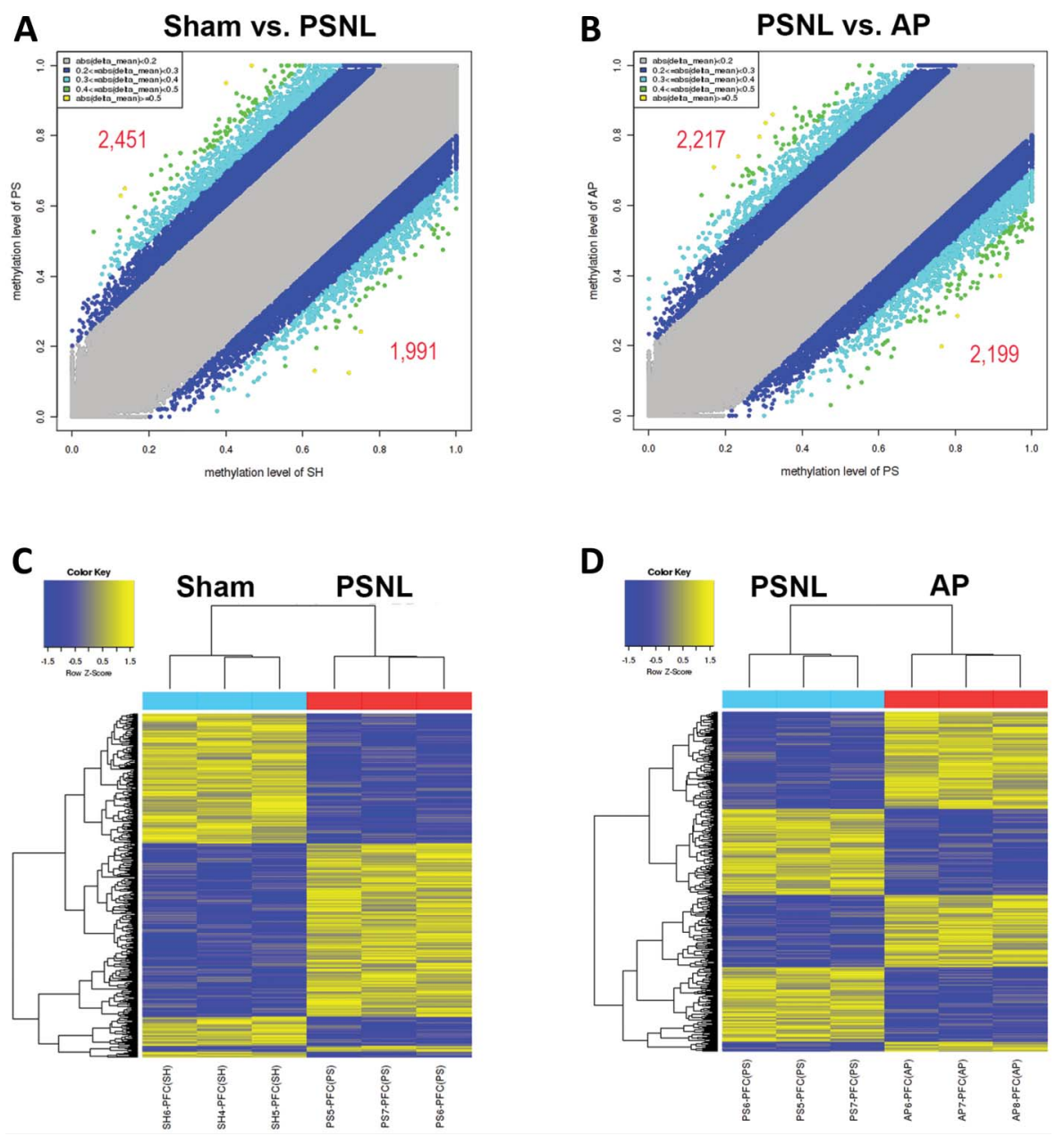

Figure 5. Effects of acupuncture on DNA methylation patterns in the PFC. WGBS was performed to identify the genes methylated by acupuncture treatment for 6 months ( $n=3$ /group). DNA methylation profiling was performed in the promoter regions based on differentially methylated regions (DMRs). Scatter plot comparisons of DMRs in the PSNL vs sham (A) and PSNL vs AP (B) groups are shown. Heat maps of methylated genes in the PSNL vs sham (C) and PSNL vs AP (D) group comparisons are shown. AP, acupuncture points; PFC, prefrontal cortex; PSNL, partial sciatic nerve ligation; WGBS, whole-genome bisulfite sequencing. 


\section{A}
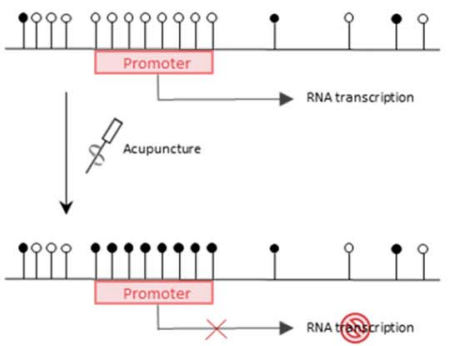

- Methylated $\mathrm{CPG}$

i Unmethylated $C P G$

C

\section{All promoters}

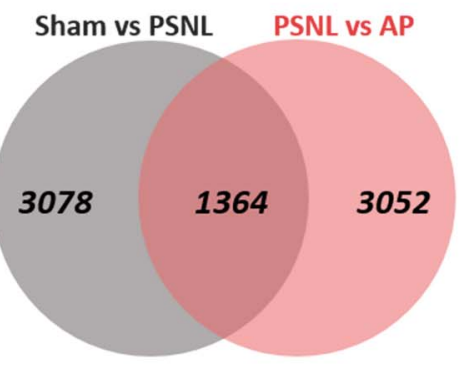

\section{E}

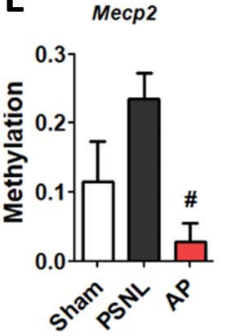

F $\quad$ Dnmt1

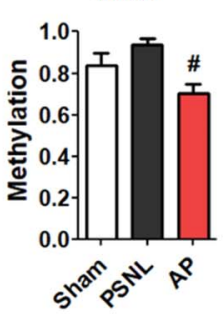

B

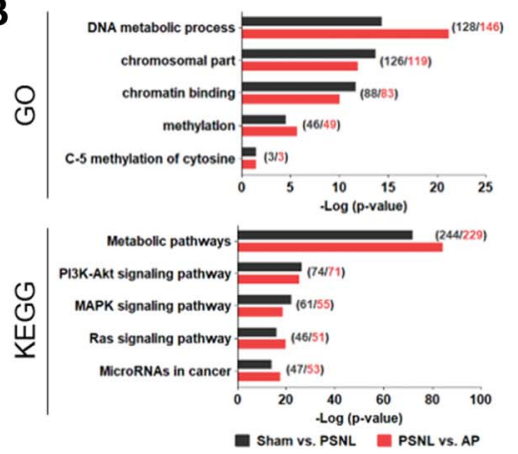

D DNA Metabolic
process 146 1364

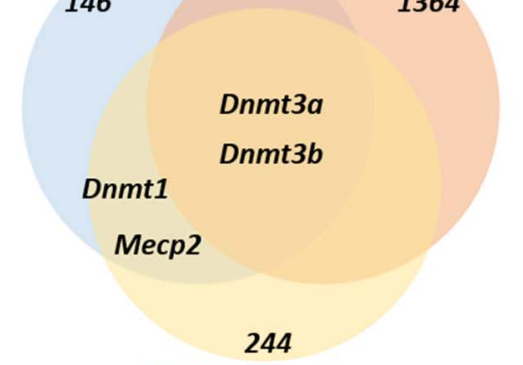

G Metabolic pathway

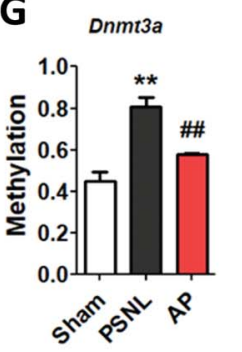

H

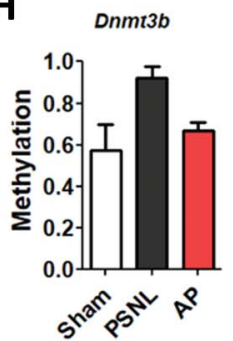

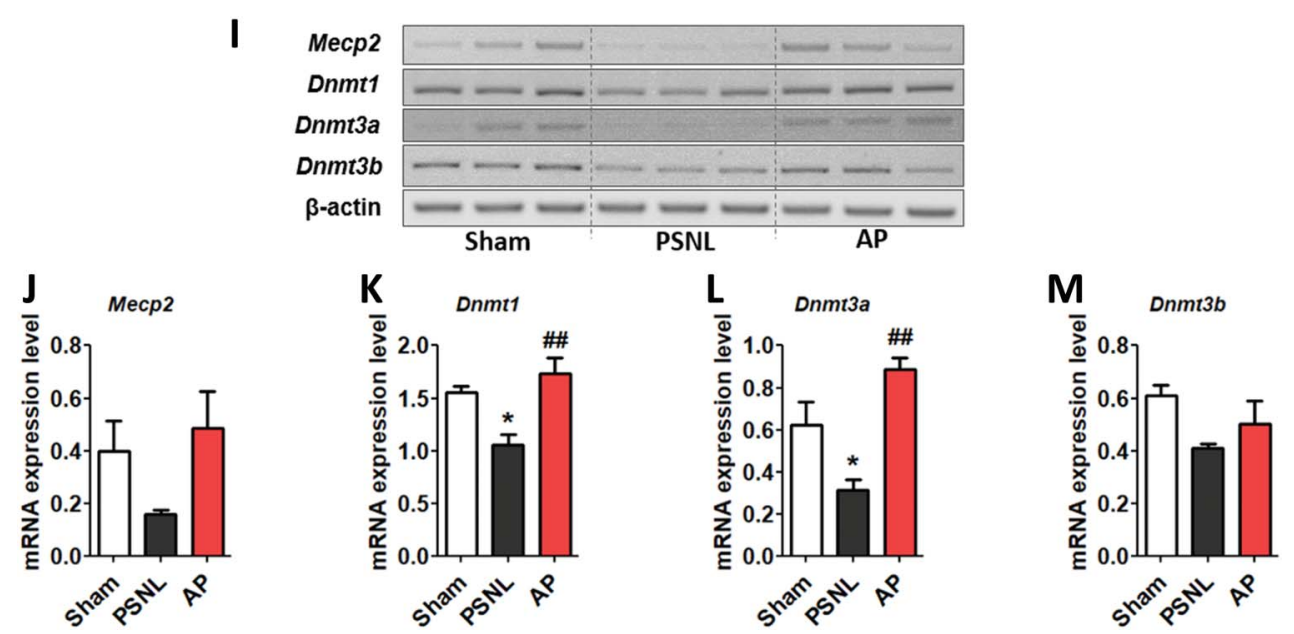

Figure 6. Effects of acupuncture on DNA methylation process-related genes in the PFC. Representative figure showing the inhibition of RNA transcription by DNA methylation in the promoter region after acupuncture treatment (A). GO and KEGG pathway enrichment methylation patterns in promoter regions. Names determined by GO analysis and KEGG pathways are shown on the left, and the bars on the right represent the -log (P-value) of the corresponding pathway (numbers in parentheses show the gene counts) (B). Venn diagram showing the distribution of overlapping genes in the promoter region in the sham vs PSNL (charcoal) and PSNL vs AP group comparisons (red) (C) and showing the shared genes among methylated genes in the DNA metabolic process and metabolic pathway (D). The graphs show changes in methylation (E-H) and mRNA expression (I-M) of Mecp2, Dnmt1, Dnmt3a, and Dnmt3b in the PFC after treatment with acupuncture (AP) for 6 months (3 days/week, $\mathrm{n}=3$ /group). ${ }^{\star} P<0.05$, ${ }^{\star *} P<0.01$ vs sham group, $\# P<0.05$, and \#\#P<0.01 vs PSNL group. The data were analyzed using one-way ANOVA followed by the Newman-Keuls post hoc test. The data are showed as the mean \pm SEM. ANOVA, analysis of variance; AP, acupuncture points; GO, Gene Ontology; KEGG, Kyoto Encyclopedia of Genes and Genomes; PFC, prefrontal cortex; PSNL, partial sciatic nerve ligation. 
5-mC (Fig. 7A) and MeCP2 (Fig. 7B) were costained with NeuN for double-immunofluorescence analysis. One-way ANOVA with the Newman-Keuls post hoc test for 5-mC- $(F[3,20]=16.07, P$ $<0.0001)$ and MeCP2-positive NeuN cells $(F[3,20]=34.71, P<$ 0.0001 ) in mice with PSNL-induced neuropathic pain demonstrated that AP treatment significantly increased global DNA methylation in the PFC (5-mC, $P<0.001$; MeCP2, $P<0.001$ vs PSNL), but DNA methylation in the PFC was not changed in the CP group compared to that in the PSNL group (Figs. 7C-D).

Next, to confirm the changes in DNMT enzymes associated with acupuncture treatment (3 days/week for 6 months) for PSNL-induced neuropathic pain, DNMT1, DNMT3a, and DNMT3b were examined by Western blot analysis in the PFC tissues of the mouse model. One-way ANOVA showed significant differences in the protein expression of DNMT1 $(F[3,12]=10.85$, $P=0.0010)$ and DNMT3a $(F[3,12]=7.601, P=0.0041)$ in the PFC among the 4 groups. The Newman-Keuls post hoc test indicated that DNMT1 and DNMT3a expression levels were significantly higher in the AP group than in the PSNL group (DNMT1, $P<0.01$; DNMT3a, $P<0.05$, respectively). The PSNL and CP groups did not show differences in DNMT1 or DNMT3a expression levels in the PFC, respectively (Figs. 7E and F). Oneway ANOVA showed a significant difference in DNMT3b expression among the groups $(F[3,12]=3.918, P=0.0366)$. The Newman-Keuls post hoc test suggested that the DNMT3b expression level was significantly decreased in the PSNL group compared to that in the sham group $(P<0.01)$, and AP treatment produced a minor improvement in DNMT3b expression (Fig. 7G).

\subsection{Acupuncture affected cell death and cell growth-related genes in the prefrontal cortex}

In 2 methylation pattern genes, hypo-hyper methylated genes (DNA methylation in the promoter of genes decreased in the PSNL group and increased in the AP group) changed by $73.53 \%$, which is more than hyper-hypo methylated genes (DNA methylation in the promoter of genes increased in the PSNL group and reduced in the AP group). We identified the top 20 genes (Nr4a1, Chkb, Tekt4, Abcb6, Chchd1, Tbx1, Aifm2, Mir7069, Fxr2, Adad1, Lypo6b, Arl8a, Rtp2, Gng7, Loxl4, Gm10432, Cpne2, Npffr1, Ttll1, and Zfp787) that were shared in the PSNL vs sham (hypomethylated genes) and PSNL vs AP (hypermethylated genes) groups (Figs. 8A and B).

Next, we determined the expression levels of Nr4a1, Chkb, Tekt4, Abcb6, Rasgrp1, and Rassf1 mRNA in hypo-hyper methylated and Ras pathway-related genes. One-way ANOVA with the Newman-Keuls post hoc test for Nr4a1 (F[2, 6] = 28.15, $P=0.0009)$, Rasgrp1 (F[2, 6] $=21.31, P=0.0019)$, Rassf1 (F[2, $6]=13.52, P=0.0060)$, Chkb $(F[2,6]=18.22, P=0.0028)$, Tekt4 (F[2, 6] $=9.661, P=0.0133)$, and Abcb6 $(\mathrm{F}[2,6]=3.840$, $P=0.0844)$ in mice with PSNL-induced neuropathic pain demonstrated that AP treatment significantly decreased the mRNA expression levels in the PFC (Nr4a1, $P<0.001$; Rasgrp1, $P<0.01$; Rassf1, $P<0.01$; Chkb, $P<0.01$; Tekt4, $P<0.01$; Abcb6, $P<0.01$ vs PSNL; Figs. 8C-I).

\subsection{Mecp2 small interfering RNA increased cell death and cell growth-related genes in the primary cortical neuron cells}

To determine the causal relationship between Mecp2 and Nr4a1, Rasgrp1, Rassf1, and Chkb, we observed the Mecp2 mRNA expression in neuronal cells treated with Mecp2 siRNA. Unpaired two-tailed $t$-tests revealed that the Mecp2 mRNA expression ( $\mathrm{t} 4=$ 9.500, $P=0.0007$ ) in Mecp2 siRNA group was significantly lower than that in the Control group $(P<0.001$; Fig. 8J). In addition, we found that the expression of Nr4a1 ( $\mathrm{t} 4=10.06, P=0.0006$ ), Rasgrp1 ( $\mathrm{t} 4=4.478, P=0.0110)$, Rassf1 ( $\mathrm{t} 4=6.685, P=0.0026)$, and Chkb (t4 $=4.234, P=0.0133$ ) was significantly increased as a result of the Mecp2 siRNA treatment (Nr4a1, $P<0.001$; Chkb, $P<$ 0.05; Rasgrp1, $P<0.05$; Rassf1, $P<0.01$ vs Control; Figs. 8K-N).

\section{Discussion}

Chronic neuropathic pain has multidimensional aspect from nociception to comorbid emotional and cognitive discomfort. Epigenetic mechanisms are involved in various comorbid conditions in neuropathic pain, and acupuncture is efficacious in such conditions. ${ }^{22,66}$ However, the role of DNA methylation in the context of acupuncture and neuropathic pain is not clear. Our results indicate that acupuncture improved nociceptive and emotional and cognitive impairment associated with PSNLinduced neuropathic pain. Acupuncture reversed the decrease in global DNA methylation by decreasing MeCP2 and DNMT enzyme levels in the PFC induced by PSNL, and restored the reduction of DNA methylation of $\mathrm{Nr} 4 \mathrm{a} 1, \mathrm{Chkb}$, and Ras pathwayrelated genes. These results demonstrate the effect of acupuncture on DNA methylation in mediating the sensory, emotional, and cognitive impact of chronic neuropathic pain, and suggested molecular mechanism in the PFC.

The central sensitization induced by peripheral nerve injury contributes to neuropathic pain, and has a pernicious effect on affective processing and cognitive function in both patients and animal models. ${ }^{46,47,54,73}$ Consistent with recent studies, ${ }^{22,66}$ PSNL mice with prolonged (6-month) mechanical hyperalgesia and cold allodynia showed both anxiety and depression in OFT, EPM, and TST tests, and working and recognition memory deficits in Y-maze and NOR tests. By contrast, acupuncture resulted in normalized emotional processing and cognitive function and allodynia. The nonacupoint (CP) group did not show these improvements, suggesting point specificity of acupoints GB30 and GB34.

DNA methylation is an essential epigenetic mechanism for regulating gene expression. ${ }^{12,24,66}$ DNA methylation plays a critical role in several physiological functions, including pain memory and synaptic plasticity. 2,22,58 Here, we observed the changes in global DNA methylation in PFC, PAG, hypothalamus, HIP, and AMG. We found that those in the PFC were the most highly correlated with both pain and comorbid behaviors compared to other brain regions. Prefrontal cortex mediates decision-making through information received from various brain areas, so it can regulate various physiological functions. ${ }^{41}$ Furthermore, PFC plays an important role in both pain and comorbidity associated with chronic pain. Decrease of cortical thickness in the PFC correlates with increase in pain intensity and pain-related disability. The PFC activity was abnormal during cognitive tasks requiring attention in the chronic low back pain patients. ${ }^{61}$ Furthermore, reduction of gray matter in the PFC was associated with the appearance of emotional dysfunction in persistent neuropathic pain rats. ${ }^{60}$ In recent studies, DNA methylation in PFC decreased in mice with persistent pain and emotional and cognitive impairment, ${ }^{22,66}$ suggesting the importance of relationship between changes in DNA methylation in PFC and comorbidity by pain. Moreover, restoration of DNA methylation levels in PFC by oral administration of $\mathrm{S}$ adenosylmethionine, a methyl donor, improves emotional and cognitive functions impaired by spared nerve injury. ${ }^{22}$ Consistent with these studies, acupuncture treatment resulted in restored global DNA methylation in PFC, indicating that the modification in 
A
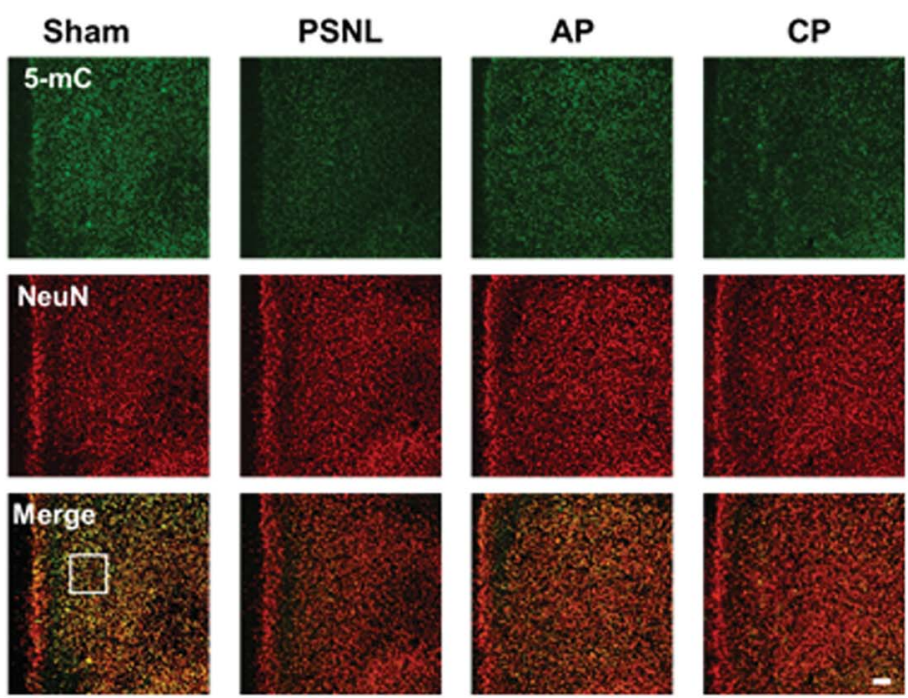

B
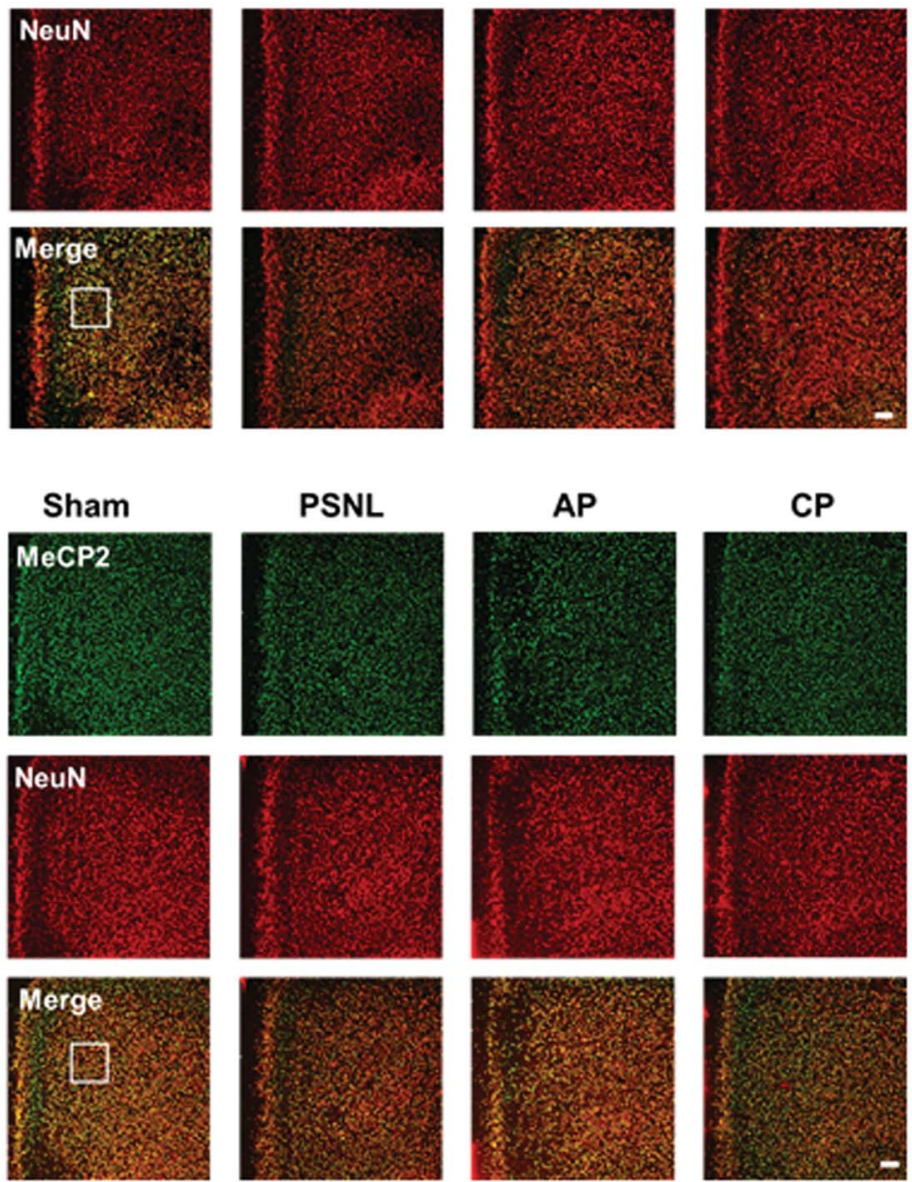

AP


E
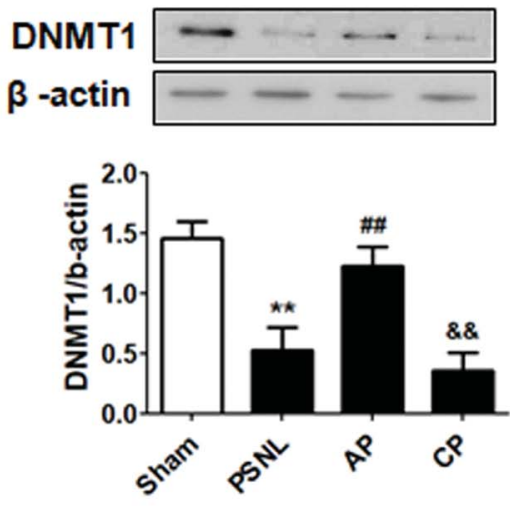

$\mathbf{F}$
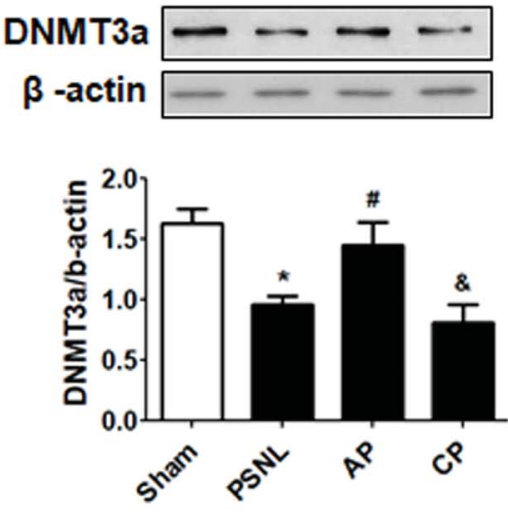

G
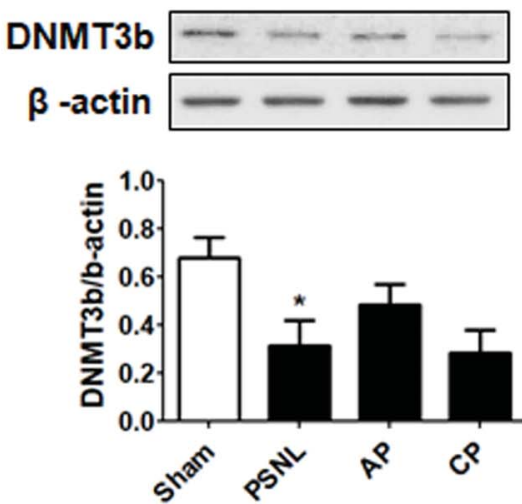

Figure 7. Effects of acupuncture on the expression levels of 5-mC, MeCP2, and DNMTs in the PFC. Histological examinations of the PFC showing expression of 5-mC (green) (A-C) and MeCP2 (green) (B-D) with NeuN (red) after acupuncture administration (AP or CP) for 6 months (3 days/week) in a PSNL-induced neuropathic pain model ( $A$ and $B$ ); this representative image shows 5-mC-positive neuron cells $(C)$ and MeCP2-positive neuron cells $(D)$ in the PFC region ( $n=6$ / group). Scale bar: $100 \mu \mathrm{m} .{ }^{\star \star \star} P<0.01$ vs sham group, \#\#\#P<0.001 vs PSNL group, and \#\#\#P<0.001 vs PSNL group. The results showed the changes in protein expression levels of DNMT1 (E), DNMT3a (F), and DNMT3b $(G)$ in the PFC after administration of AP or CP for 6 months $\left(n=4 /\right.$ group, ${ }^{\star} P<0.05,{ }^{* *} P<0.01$ vs sham group, $\# P<0.05$, \#\#P<0.01 vs PSNL group, and $\& P<0.05, \& \& P<0.01$ vs AP group). The results were analyzed using one-way ANOVA followed by the Newman-Keuls post hoc test. All data are expressed as the mean \pm SEM. 5-mC, 5-methylcytosine; ANOVA, analysis of variance; AP, acupuncture points; CP, control point; CpG, cytosine-phospho-guanine; DNMT, DNA methyltransferase; MeCP2, methyl-CpG binding protein 2; PFC, prefrontal cortex.

DNA methylation might be a promising therapeutic target for acupuncture treatment.

We showed that in the genome-wide methylation patterns in the PFC associated with neuropathic pain and acupuncture, the top 5 GO terms were DNA metabolic process, chromosomal part, chromatin binding, methylation, and C-5 methylation of cytosine using $\mathrm{GO}$ analysis, and the top 5 KEGG pathways were metabolic pathways, PI3K-Akt signaling pathway, MAPK kinase signaling pathway, Ras signaling pathway, and microRNAs in cancer. Among genes altered by acupuncture treatment in the promoter 


\section{A}

B


I
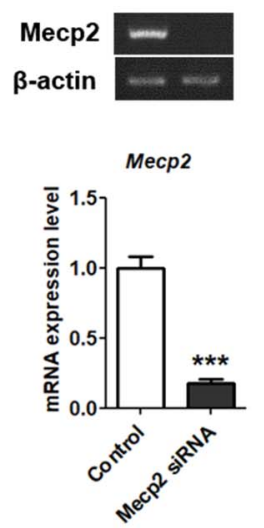

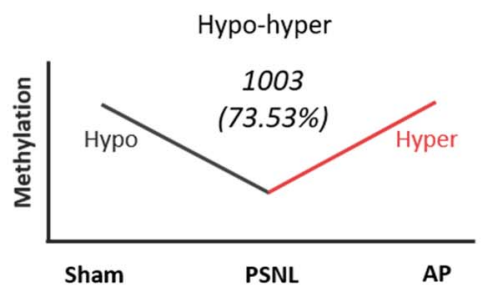

$\mathbf{F}$

C


$\square$ SH PS
J

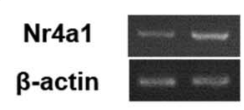

Nr4a1

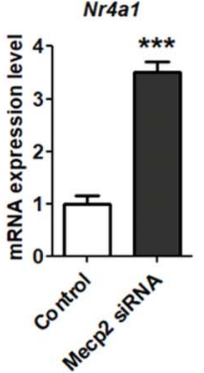

D

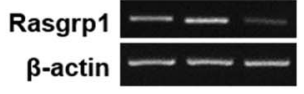

G



L
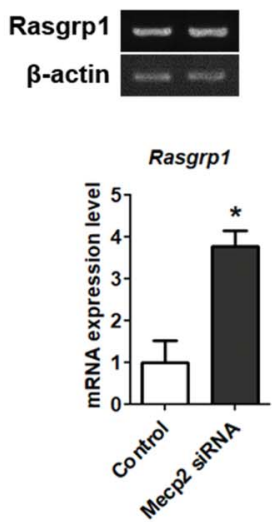
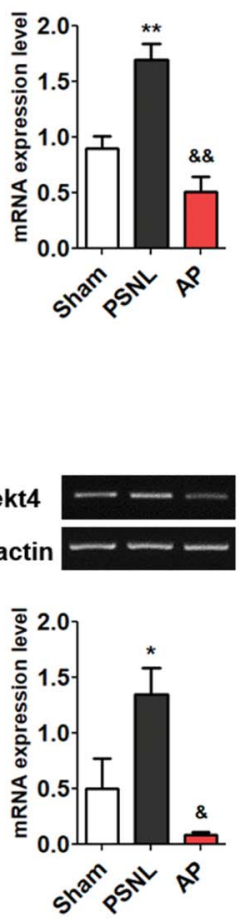

E
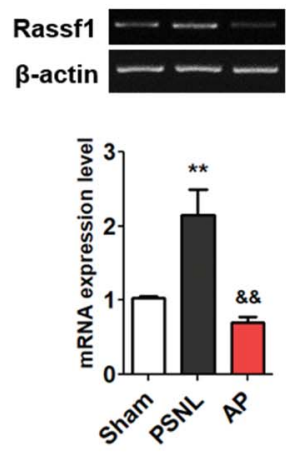

H
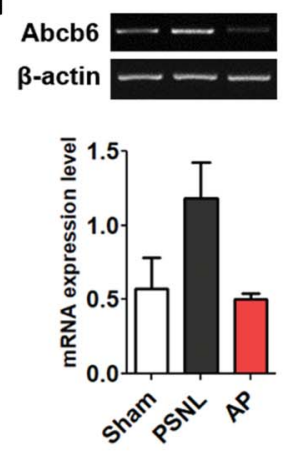

M

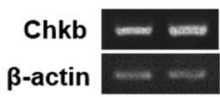

Rassf1

$\beta$-actin $\cdots$

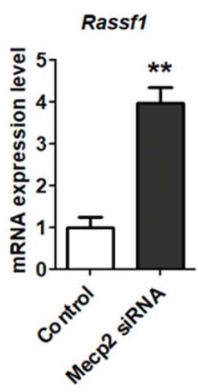

Chkb

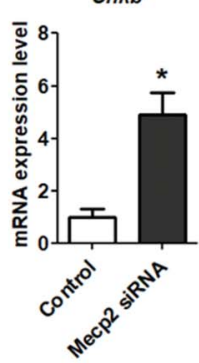

Figure 8. Effects of acupuncture on the methylation levels of cell death-related genes in the PFC. The graphs showing the genes of the hypo-hyper methylation pattern (A) and top 20 in the hypo-hyper methylated genes (B) in the PFC. The graphs show the changes in the mRNA expression of Nr4a1, Rasgrp1, Rassf1, Chkb, Tekt4, and Abcb6 (C-H) in the PFC after administration of AP or CP for 6 months. The graphs show changes in the mRNA expression of Mecp2, Dnmt1, Dnmt3a, and Dnmt3b at 3 days after treatment with Mecp2 siRNA in the neuronal cell of the mouse frontal cortex $(1-\mathrm{M}) . \mathrm{n}=3 /$ group, ${ }^{*} P<0.05$, ${ }^{* *} P<0.01$ vs sham group, $\# P<0.05$, \#\#P<0.01, \#\#\#P<0.001 vs PSNL group. All data were analyzed using one-way ANOVA followed by the Newman-Keuls post hoc test. All data are shown as mean \pm SEM. ANOVA, analysis of variance; AP, acupuncture points; CP, control point; PFC, prefrontal cortex; PSNL, partial sciatic nerve ligation; siRNA, small interfering RNA.

region, Mecp2, dnmt1, dnmt3a, and dnmt3b belonged to DNA metabolic process and metabolic pathways, and these genes are involved in DNA methylation. ${ }^{12,24,44}$ MeCP2 null-mice used in the Rett syndrome model exhibit psychomotor and intellectual disability, and overall metabolic imbalances (eg, mitochondrial dysfunction). ${ }^{52,53}$ Various types of neuropathic pain induce a reduction in metabolic activity and lead to metabolic imbalances such as mitochondrial dysfunction in DRG neurons. ${ }^{18,74}$ Consistent with previous studies, PSNL mice showed decreased 5-mCand MeCP2-positive neurons and reduced DNMT1 and DNMT3a
mRNA/protein expression in PFC. Maltreatment in adult male mice causes decrease in Mecp2, Dnmt1, and Dnmt3a mRNA levels in the medial PFC, ${ }^{4}$ and MeCP2 null mice produces functional hypoconnectivity in PFC that induces abnormal behavior. ${ }^{59}$ Interestingly, in PFC of mice with chronic stress, knockdown of Dnmt3a mRNA induces anxiety-like behavior, which is inhibited by overexpressed Dnmt3a mRNA. ${ }^{14}$ In line with these results, acupuncture increased MeCP2, DNMT1, and DNMT3a mRNA/protein expression in the PFC, which may be important for behavior normalization. Thus, we suggest that 
neuropathic pain increases DNA methylation in Mecp2, Dnmt1, and Dnmt3a in the PFC, which contributes to reduction in global DNA methylation in PFC and induces various abnormal behaviors, which are restored by acupuncture.

We identified genes with reduced DNA methylation in the PSNL mice and genes with increased DNA methylation in acupuncturetreated PSNL mice compared to the PSNL mice. The ratio of hypo-hyper methylated genes was $73.53 \%$ in genes with a hypohyper and hyper-hypo methylation pattern, and we sorted top 20 in genes with the hypo-hyper methylation pattern. Among the hypo-hyper methylated genes, $\mathrm{Nr} 4 \mathrm{a} 1$ is associated with the Ras pathway ${ }^{10,21}$ and is involved in mitochondrial dysfunction and cell death in preclinical studies. ${ }^{79} \mathrm{Nr} 4 \mathrm{a} 1$ is inhibited by knockdown of Rasd1 in AtT20 cells. ${ }^{21}$ Rasgrp1 mRNA expression increases in Nr4a1-enhanced GFP mice compared with the wild-type mice. ${ }^{10}$ Interestingly, Nr4a1 mRNA expression increases in the brain of patients with major depressive disorders and Alzheimer disease. ${ }^{28}$ Activation of NR4A1 after chronic stress induces reduction in dendritic spine number and mitochondrial function in PFC of mice. ${ }^{28}$ Here, we demonstrated that increased $\mathrm{Nr} 4 a 1$ mRNA expression in the PSNL mice decreased after acupuncture treatment. In addition, to confirm whether the Ras pathway is actually involved, we examined its mRNA expression and found that the expressions of Rasgrp1 and Rassf1 mRNA were increased in the PSNL mice, and restored by acupuncture treatment. Rasgrp1 increases the activation of Rassf1 by converting RasGDP to RasGTP, which in turn activates the Ras pathway. ${ }^{30,56} \mathrm{~A}$ Ras family member enriched in brain is increased in activated astrocytes by injured spinal cord. ${ }^{48}$ Previous studies showed that Ras pathway plays important role in regulation of mitochondrial function in the brain. ${ }^{39,65}$ We found that DNA methylation of Chkb (associated with mitochondrial dysfunction and cell death) was restored after acupuncture treatment in the PSNL mice. Activation of Chkb increases mitochondrial stress signaling and oxidative stress in vivo and in vitro. ${ }^{5}$ We also showed its increased mRNA expression in the PSNL mice was reduced after acupuncture treatment. Finally, we tested the changes in gene expression after Mecp2 siRNA treatment in neuronal cells of the frontal cortex, and found that the mRNA levels of Nr4a1, Rasgrp1, Rassf1, and Chkb were increased, similar to previous results. ${ }^{67}$ Thus, we suggest that acupuncture may improve the pain symptoms induced through increase in Ras pathway- and mitochondrial function-related genes by reducing DNA methylation in PFC of neuropathic pain. However, the role of Ras pathway and mitochondrial function in the PFC of neuropathic pain is still unknown, and further research is needed.

Because chronic pain induces overall structural and functional changes in the CNS, alterations in DNA methylation in other brain regions must be investigated. Here, we observed global DNA methylation alterations in the HIP, AMG, PAG, and hypothalamus, which are important regions for pain sensitivity and comorbidities in chronic pain. ${ }^{3,8,19,66,77}$ We found that global DNA methylation in PSNL mice decreased in PAG and increased in HIP, which correlated with behavioral changes. Acupuncture treatments significantly decreased global DNA methylation, which was increased by PSNL in the HIP. To the best of our knowledge, this is the first study showing an association between global DNA methylation in the PAG and HIP of a PSNL model; further studies are warranted to elucidate the exact mechanism of action.

Our study has several limitations. First, the causal relationship between DNA methylation changes in the brain and comorbidity with neuropathic pain or the effect of acupuncture was not defined. Whether acupuncture directly increased the global DNA methylation in the PFC is unclear because the experiments on the effect of acupuncture using siRNA or an antagonist were not conducted. Future studies should establish the causal relationship by inhibiting the effects of acupuncture on DNA methylation in neuropathic pain. In our results, MAPK and PI3K-Akt signaling pathway-related genes such as Mapkapk3, Bc/2/11, and Bdnf showed decrease in DNA methylation after acupuncture treatment in PSNL mice, indicating a hyper-hypo methylation pattern (26.47\%). These pathway genes play important roles in the analgesic effect of acupuncture. ${ }^{34,36,69}$ Thus, further studies are needed on the MAPK and PI3K-Akt signaling pathways and genes with hyper-hypo methylation patterns, including Mapkapk3, Bc/2/11, and Bdnf.

Our results are consistent with the hypothesis that acupuncture alters the extent of DNA methylation induced by neuropathic pain. Peripheral nerve injury, which induces changes in global DNA methylation in the PFC, was reversed using acupuncture. Our results also indicate that modulation of DNA methylation- and mitochondrial dysfunction-related genes may play a key role in the acupuncture-induced reversal of physiological function impairment caused by persistent neuropathic pain. Our findings enhance the molecular understanding of neuropathic pain, and may facilitate the discovery of new mechanisms of action in acupuncture treatment.

\section{Conflict of interest statement}

The authors have no conflicts of interest to declare.

\section{Acknowledgements}

This research was supported by grants from the National Research Foundation of Korea funded by the Korean government (NRF-2017R1A2B4009963 and NRF-2017R1A2B4011707) and from the Korea Institute of Oriental Medicine (grant K18182). All other authors declare no competing financial interests.

\section{Appendix A. Supplemental digital content}

Supplemental digital content associated with this article can be found online at http://links.Iww.com/PAIN/B143.

\section{Article history:}

Received 30 March 2020

Received in revised form 7 July 2020

Accepted 13 July 2020

Available online 6 August 2020

\section{References}

[1] Alvarado S, Tajerian M, Millecamps M, Suderman M, Stone LS, Szyf M. Peripheral nerve injury is accompanied by chronic transcriptome-wide changes in the mouse prefrontal cortex. Mol Pain 2013;9:21.

[2] Alvarado S, Tajerian M, Suderman M, Machnes Z, Pierfelice S, Millecamps M, Stone LS, Szyf M. An epigenetic hypothesis for the genomic memory of pain. Front Cell Neurosci 2015;9:88.

[3] Bilbao A, Falfan-Melgoza C, Leixner S, Becker R, Singaravelu SK, Sack M, Sartorius A, Spanagel R, Weber-Fahr W. Longitudinal structural and functional brain network alterations in a mouse model of neuropathic pain. Neuroscience 2018;387:104-15.

[4] Blaze J, Roth TL. Exposure to caregiver maltreatment alters expression levels of epigenetic regulators in the medial prefrontal cortex. Int J Dev Neurosci 2013;31:804-10.

[5] Brady L, Giri M, Provias J, Hoffman E, Tarnopolsky M. Proximal myopathy with focal depletion of mitochondria and megaconial congenital muscular dystrophy are allelic conditions caused by mutations in CHKB. Neuromuscul Disord 2016;26:160-4. 
[6] Bushnell MC, Ceko M, Low LA. Cognitive and emotional control of pain and its disruption in chronic pain. Nat Rev Neurosci 2013;14:502-11.

[7] Chen RW, Liu H, An JX, Qian XY, Jiang YD, Cope DK, Williams JP, Zhang R, Sun LN. Cognitive effects of electro-acupuncture and pregabalin in a trigeminal neuralgia rat model induced by cobra venom. J Pain Res 2017; 10:1887-97.

[8] Cheriyan J, Sheets PL. Altered excitability and local connectivity of mPFCPAG neurons in a mouse model of neuropathic pain. J Neurosci 2018;38: 4829-39.

[9] Cho YJ, Song YK, Cha YY, Shin BC, Shin IH, Park HJ, Lee HS, Kim KW, Cho JH, Chung WS, Lee JH, Song MY. Acupuncture for chronic low back pain: a multicenter, randomized, patient-assessor blind, sham-controlled clinical trial. Spine 2013;38:549-57.

[10] Cirnaru MD, Melis C, Fanutza T, Naphade S, Tshilenge KT, Muntean BS, Martemyanov KA, Plotkin JL, Ellerby LM, Ehrlich ME. Nuclear receptor $\mathrm{Nr} 4 \mathrm{a} 1$ regulates striatal striosome development and dopamine D1 receptor signaling. eNeuro 2019;6. doi: 10.1523/ENEURO.030519.2019.

[11] Cui P, Ma T, Tamadon A, Han S, Li B, Chen Z, An X, Shao LR, Wang Y, Feng $Y$. Hypothalamic DNA methylation in rats with dihydrotestosteroneinduced polycystic ovary syndrome: effects of low-frequency electroacupuncture. Exp Physiol 2018;103:1618-32.

[12] Denk F, McMahon SB. Chronic pain: emerging evidence for the involvement of epigenetics. Neuron 2012;73:435-44.

[13] Descalzi G, Ikegami D, Ushijima T, Nestler EJ, Zachariou V, Narita M. Epigenetic mechanisms of chronic pain. Trends Neurosci 2015;38: 237-46.

[14] Elliott E, Manashirov S, Zwang R, Gil S, Tsoory M, Shemesh Y, Chen A. Dnmt3a in the medial prefrontal cortex regulates anxiety-like behavior in adult mice. J Neurosci 2016;36:730-40.

[15] Feng J, Chang H, Li E, Fan G. Dynamic expression of de novo DNA methyltransferases Dnmt3a and Dnmt3b in the central nervous system. J Neurosci Res 2005;79:734-46.

[16] Feng J, Zhou Y, Campbell SL, Le T, Li E, Sweatt JD, Silva AJ, Fan G. Dnmt1 and Dnmt3a maintain DNA methylation and regulate synaptic function in adult forebrain neurons. Nat Neurosci 2010;13:423-30.

[17] Frymoyer JW. Back pain and sciatica. N Engl J Med 1988;318:291-300.

[18] Galley HF, McCormick B, Wilson KL, Lowes DA, Colvin L, Torsney C. Melatonin limits paclitaxel-induced mitochondrial dysfunction in vitro and protects against paclitaxel-induced neuropathic pain in the rat. J Pineal Res 2017;63:e12444.

[19] Gao Y, Chen S, Xu Q, Yu K, Wang J, Qiao L, Meng F, Liu J. Proteomic analysis of differential proteins related to anti-nociceptive effect of electroacupuncture in the hypothalamus following neuropathic pain in rats. Neurochem Res 2013;38:1467-78.

[20] Gao YH, Li CW, Wang JY, Kan Y, Tan LH, Jing XH, Liu JL. Activation of hippocampal MEK1 contributes to the cumulative antinociceptive effect of electroacupuncture in neuropathic pain rats. BMC Complement Altern Med 2016;16:517

[21] Greenwood MP, Greenwood M, Mecawi AS, Antunes-Rodrigues J, Paton JF, Murphy D. Rasd1, a small G protein with a big role in the hypothalamic response to neuronal activation. Mol Brain 2016;9:1.

[22] Gregoire S, Millecamps M, Naso L, Do Carmo S, Cuello AC, Szyf M, Stone LS. Therapeutic benefits of the methyl donor Sadenosylmethionine on nerve injury-induced mechanical hypersensitivity and cognitive impairment in mice. PAIN 2017;158: 802-10.

[23] Gui WS, Wei X, Mai CL, Murugan M, Wu LJ, Xin WJ, Zhou LJ, Liu XG. Interleukin-1beta overproduction is a common cause for neuropathic pain, memory deficit, and depression following peripheral nerve injury in rodents. Mol Pain 2016;12:1744806916646784.

[24] Guo JU, Ma DK, Mo H, Ball MP, Jang MH, Bonaguidi MA, Balazer JA, Eaves HL, Xie B, Ford E, Zhang K, Ming GL, Gao Y, Song H. Neuronal activity modifies the DNA methylation landscape in the adult brain. Nat Neurosci 2011;14:1345-51.

[25] Hopton A, Macpherson H, Keding A, Morley S. Acupuncture, counselling or usual care for depression and comorbid pain: secondary analysis of a randomised controlled trial. BMJ Open 2014;4:e004964.

[26] Jang JH, Kim YK, Jung WM, Kim HK, Song EM, Kim HY, Oh JY, Park JY, Ryu Y, Song MY, Park HJ. Acupuncture improves comorbid cognitive impairments induced by neuropathic pain in mice. Front Neurosci 2019; 13:995.

[27] Jang JH, Park JY, Oh JY, Bae SJ, Jang H, Jeon S, Kim J, Park HJ. Novel analgesic effects of melanin-concentrating hormone on persistent neuropathic and inflammatory pain in mice. Sci Rep 2018;8:707.

[28] Jeanneteau F, Barrere C, Vos M, De Vries CJM, Rouillard C, Levesque D, Dromard Y, Moisan MP, Duric V, Franklin TC, Duman RS, Lewis DA, Ginsberg SD, Arango-Lievano M. The stress-induced transcription factor
NR4A1 adjusts mitochondrial function and synapse number in prefrontal cortex. J Neurosci 2018;38:1335-50.

[29] Jones PL, Veenstra GJ, Wade PA, Vermaak D, Kass SU, Landsberger N, Strouboulis J, Wolffe AP. Methylated DNA and MeCP2 recruit histone deacetylase to repress transcription. Nat Genet 1998;19:187-91.

[30] Khokhlatchev A, Rabizadeh S, Xavier R, Nedwidek M, Chen T, Zhang XF, Seed B, Avruch J. Identification of a novel Ras-regulated proapoptotic pathway. Curr Biol 2002;12:253-65.

[31] Kim KW, Park K, Park HJ, Jahng GH, Jo DJ, Cho JH, Song EM, Shin WC, Yoon YJ, Kim SJ, Eun S, Song MY. Effect and neurophysiological mechanism of acupuncture in patients with chronic sciatica: protocol for a randomized, patient-assessor blind, sham-controlled clinical trial. Trials 2019;20:56.

[32] Kim SN, Kim ST, Doo AR, Park JY, Moon W, Chae Y, Yin CS, Lee H, Park HJ. Phosphatidylinositol 3-kinase/Akt signaling pathway mediates acupuncture-induced dopaminergic neuron protection and motor function improvement in a mouse model of Parkinson's disease. Int J Neurosci 2011;121:562-9.

[33] Liang L, Lutz BM, Bekker A, Tao YX. Epigenetic regulation of chronic pain. Epigenomics 2015;7:235-45.

[34] Liang Y, Du JY, Qiu YJ, Fang JF, Liu J, Fang JQ. Electroacupuncture attenuates spinal nerve ligation-induced microglial activation mediated by p38 mitogen-activated protein kinase. Chin J Integr Med 2016;22: 704-13.

[35] Ligon CO, Moloney RD, Greenwood-Van Meerveld B. Targeting epigenetic mechanisms for chronic pain: a valid approach for the development of novel therapeutics. J Pharmacol Exp Ther 2016;357: 84-93.

[36] Liu J, Wu Y. Electro-acupuncture-modulated miR-214 prevents neuronal apoptosis by targeting Bax and inhibits sodium channel Nav1.3 expression in rats after spinal cord injury. Biomed Pharmacother 2017 ; 89:1125-35.

[37] Liu QF, Jeong H, Lee JH, Hong YK, Oh Y, Kim YM, Suh YS, Bang S, Yun HS, Lee K, Cho SM, Lee SB, Jeon S, Chin YW, Koo BS, Cho KS. Coriandrum sativum suppresses abeta42-induced ROS increases, glial cell proliferation, and ERK activation. Am J Chin Med 2016;44:1325-47.

[38] Loeser D. Bonica's Management of Pain. (3rd ed). Lippincott Williams \& Wilkins, 2001. pp. 17-25.

[39] Lu J, Wu DM, Zheng YL, Hu B, Cheng W, Zhang ZF, Li MQ. Troxerutin counteracts domoic acid-induced memory deficits in mice by inhibiting CCAAT/enhancer binding protein beta-mediated inflammatory response and oxidative stress. J Immunol 2013;190:3466-79.

[40] Malmberg AB, Basbaum Al. Partial sciatic nerve injury in the mouse as a model of neuropathic pain: behavioral and neuroanatomical correlates. PAIN 1998;76:215-22.

[41] Manes F, Sahakian B, Clark L, Rogers R, Antoun N, Aitken M, Robbins T. Decision-making processes following damage to the prefrontal cortex. Brain 2002;125(pt 3):624-39.

[42] McGowan PO, Sasaki A, D’Alessio AC, Dymov S, Labonte B, Szyf M, Turecki G, Meaney MJ. Epigenetic regulation of the glucocorticoid receptor in human brain associates with childhood abuse. Nat Neurosci 2009;12:342-8.

[43] McGowan PO, Sasaki A, Huang TC, Unterberger A, Suderman M, Ernst C, Meaney MJ, Turecki G, Szyf M. Promoter-wide hypermethylation of the ribosomal RNA gene promoter in the suicide brain. PLoS One 2008;3: e2085.

[44] Moore LD, Le T, Fan G. DNA methylation and its basic function. Neuropsychopharmacology 2013;38:23-38.

[45] Murgatroyd C, Patchev AV, Wu Y, Micale V, Bockmuhl Y, Fischer D, Holsboer F, Wotjak CT, Almeida OF, Spengler D. Dynamic DNA methylation programs persistent adverse effects of early-life stress. Nat Neurosci 2009;12:1559-66.

[46] Mutso AA, Radzicki D, Baliki MN, Huang L, Banisadr G, Centeno MV, Radulovic J, Martina M, Miller RJ, Apkarian AV. Abnormalities in hippocampal functioning with persistent pain. J Neurosci 2012;32: 5747-56.

[47] Nicholson B, Verma S. Comorbidities in chronic neuropathic pain. Pain Med 2004;5(suppl 1):S9-S27.

[48] Norsted Gregory E, Codeluppi S, Gregory JA, Steinauer J, Svensson Cl. Mammalian target of rapamycin in spinal cord neurons mediates hypersensitivity induced by peripheral inflammation. Neuroscience 2010;169:1392-402.

[49] Palazzo E, Luongo L, Guida F, Marabese I, Romano R, lannotta M, Ross F, D'Aniello A, Stella L, Marmo F, Usiello A, de Bartolomeis A, Maione S, de Novellis V. D-Aspartate drinking solution alleviates pain and cognitive impairment in neuropathic mice. Amino Acids 2016;48:1553-67.

[50] Park JY, Kim SN, Yoo J, Jang J, Lee A, Oh JY, Kim H, Oh ST, Park SU, Kim J, Park HJ, Jeon S. Novel neuroprotective effects of melanin- 
concentrating hormone in Parkinson's disease. Mol Neurobiol 2017;54: 7706-21.

[51] Park JY, Park JJ, Jeon S, Doo AR, Kim SN, Lee H, Chae Y, Maixner W, Lee $\mathrm{H}$, Park HJ. From peripheral to central: the role of ERK signaling pathway in acupuncture analgesia. J Pain 2014;15:535-49.

[52] Park MJ, Aja S, Li Q, Degano AL, Penati J, Zhuo J, Roe CR, Ronnett GV. Anaplerotic triheptanoin diet enhances mitochondrial substrate use to remodel the metabolome and improve lifespan, motor function, and sociability in MeCP2-null mice. PLoS One 2014;9:e109527.

[53] Pratte M, Panayotis N, Ghata A, Villard L, Roux JC. Progressive motor and respiratory metabolism deficits in post-weaning Mecp2-null male mice. Behav Brain Res 2011;216:313-20.

[54] Price DD. Psychological and neural mechanisms of the affective dimension of pain. Science 2000;288:1769-72.

[55] Rodriguez-Raecke R, Niemeier A, Ihle K, Ruether W, May A. Brain gray matter decrease in chronic pain is the consequence and not the cause of pain. J Neurosci 2009;29:13746-50.

[56] Roose JP, Mollenauer M, Ho M, Kurosaki T, Weiss A. Unusual interplay of two types of Ras activators, RasGRP and SOS, establishes sensitive and robust Ras activation in lymphocytes. Mol Cell Biol 2007;27:2732-45.

[57] Ropper AH, Zafonte RD. Sciatica. N Engl J Med 2015;372:1240-8.

[58] Roth TL, Sweatt JD. Regulation of chromatin structure in memory formation. Curr Opin Neurobiol 2009;19:336-42.

[59] Sceniak MP, Lang M, Enomoto AC, James Howell C, Hermes DJ, Katz DM. Mechanisms of functional hypoconnectivity in the medial prefrontal cortex of Mecp2 null mice. Cereb Cortex 2016;26:1938-56.

[60] Seminowicz DA, Laferriere AL, Millecamps M, Yu JS, Coderre TJ, Bushnell MC. MRI structural brain changes associated with sensory and emotional function in a rat model of long-term neuropathic pain. Neurolmage 2009;47:1007-14.

[61] Seminowicz DA, Wideman TH, Naso L, Hatami-Khoroushahi Z, Fallatah S, Ware MA, Jarzem P, Bushnell MC, Shir Y, Ouellet JA, Stone LS. Effective treatment of chronic low back pain in humans reverses abnormal brain anatomy and function. J Neurosci 2011;31:7540-50.

[62] Seo SY, Lee KB, Shin JS, Lee J, Kim MR, Ha $I$, Ko Y, Lee YJ. Effectiveness of acupuncture and electroacupuncture for chronic neck pain: a systematic review and meta-analysis. Am J Chin Med 2017;45: 1573-95.

[63] Sniezek DP, Siddiqui IJ. Acupuncture for treating anxiety and depression in women: a clinical systematic review. Med Acupunct 2013;25:164-72.

[64] Suri P, Palmer MR, Tsepilov YA, Freidin MB, Boer CG, Yau MS, Evans DS, Gelemanovic A, Bartz TM, Nethander M, Arbeeva L, Karssen L, Neogi T, Campbell A, Mellstrom D, Ohlsson C, Marshall LM, Orwoll E, Uitterlinden A, Rotter JI, Lauc G, Psaty BM, Karlsson MK, Lane NE, Jarvik GP, Polasek O, Hochberg M, Jordan JM, Van Meurs JBJ, Jackson R, Nielson CM, Mitchell BD, Smith BH, Hayward C, Smith NL, Aulchenko YS, Williams FMK. Genome-wide meta-analysis of 158,000 individuals of European ancestry identifies three loci associated with chronic back pain. PLoS Genet 2018;14:e1007601.
[65] Szatmari EM, Oliveira AF, Sumner EJ, Yasuda R. Centaurin-alpha1-RasElk-1 signaling at mitochondria mediates beta-amyloid-induced synaptic dysfunction. J Neurosci 2013;33:5367-74.

[66] Tajerian M, Alvarado S, Millecamps M, Vachon P, Crosby C, Bushnell MC, Szyf M, Stone LS. Peripheral nerve injury is associated with chronic, reversible changes in global DNA methylation in the mouse prefrontal cortex. PLoS One 2013;8:e55259.

[67] Tao H, Tao JY, Song ZY, Shi P, Wang Q, Deng ZY, Ding XS. MeCP2 triggers diabetic cardiomyopathy and cardiac fibroblast proliferation by inhibiting RASSF1A. Cell Signal 2019;63:109387.

[68] Treede RD, Rief W, Barke A, Aziz Q, Bennett MI, Benoliel R, Cohen M, Evers S, Finnerup NB, First MB, Giamberardino MA, Kaasa S, Kosek E, Lavand'homme P, Nicholas M, Perrot S, Scholz J, Schug S, Smith BH, Svensson P, Vlaeyen JW, Wang SJ. A classification of chronic pain for ICD-11. PAIN 2015;156:1003-7.

[69] Tu WZ, Li SS, Jiang X, Qian XR, Yang GH, Gu PP, Lu B, Jiang SH. Effect of electro-acupuncture on the BDNF-TrkB pathway in the spinal cord of $\mathrm{CCl}$ rats. Int J Mol Med 2018;41:3307-15.

[70] Vickers AJ, Vertosick EA, Lewith G, MacPherson H, Foster NE, Sherman KJ, Irnich D, Witt CM, Linde K, Acupuncture Trialists C. Acupuncture for chronic pain: update of an individual patient data meta-analysis. J Pain 2018;19:455-74.

[71] Woo AK. Depression and anxiety in pain. Rev Pain 2010;4:8-12.

[72] Xi Y, Li W. BSMAP: whole genome bisulfite sequence MAPping program. BMC Bioinformatics 2009;10:232.

[73] Yalcin I, Barthas F, Barrot M. Emotional consequences of neuropathic pain: insight from preclinical studies. Neurosci Biobehav Rev 2014;47:154-64.

[74] Yamashita A, Matsuoka Y, Matsuda M, Kawai K, Sawa T, Amaya F. Dysregulation of p53 and parkin induce mitochondrial dysfunction and leads to the diabetic neuropathic pain. Neuroscience 2019;416:9-19.

[75] Yu Y, Mo Y, Ebenezer D, Bhattacharyya S, Liu H, Sundaravel S, Giricz O, Wontakal S, Cartier J, Caces B, Artz A, Nischal S, Bhagat T, Bathon K, Maqbool S, Gligich O, Suzuki M, Steidl U, Godley L, Skoultchi A, Greally J, Wickrema A, Verma A. High resolution methylome analysis reveals widespread functional hypomethylation during adult human erythropoiesis. J Biol Chem 2013;288:8805-14.

[76] Zhang XG, Zhang H, Liang XL, Liu Q, Wang HY, Cao B, Cao J, Liu S, Long YJ, Xie WY, Peng DZ. Epigenetic mechanism of maternal post-traumatic stress disorder in delayed rat offspring development: dysregulation of methylation and gene expression. Genet Mol Res 2016;15:15039009.

[77] Zhang Y, Mao Z, Pan L, Ling Z, Liu X, Zhang J, Yu X. Dysregulation of pain- and emotion-related networks in trigeminal neuralgia. Front Hum Neurosci 2018;12:107.

[78] Zhou J, Peng W, Xu M, Li W, Liu Z. The effectiveness and safety of acupuncture for patients with Alzheimer disease: a systematic review and meta-analysis of randomized controlled trials. Medicine 2015;94:e933.

[79] Zong C, Qin D, Yu C, Gao P, Chen J, Lu S, Zhang Y, Liu Y, Yang Y, Pu Z, Li X, Fu Y, Guan Q, Wang X. The stress-response molecule NR4A1 resists ROSinduced pancreatic beta-cells apoptosis via WT1. Cell Signal 2017;35:129-39. 